\title{
Stationary Two-Dimensional Magnetohydrodynamic Flows with Shocks: Characteristic Analysis and Grid Convergence Study
}

\author{
H. De Sterck, ${ }^{*, 1}$ A. Csík, ${ }^{*}$, D. Vanden Abeele, $\nmid$ S. Poedts, ${ }^{*, 2}$ and H. Deconinck $\dagger$
}

*Centre for Plasma Astrophysics, K.U. Leuven, Celestijnenlaan 200B, 3001 Leuven, Belgium; and †von Karman Institute for Fluid Dynamics, Waterloose Steenweg 72, 1640 Sint-Genesius-Rode, Belgium

E-mail: hans.desterck@wis.kuleuven.ac.be

Received June 18, 1999

Five model flows of increasing complexity belonging to the class of stationary two-dimensional planar field-aligned magnetohydrodynamic (MHD) flows are presented which are well suited to the quantitative evaluation of MHD codes. The physical properties of these five flows are investigated using characteristic theory. Grid convergence criteria for flows belonging to this class are derived from characteristic theory, and grid convergence is demonstrated for the numerical simulation of the five model flows with a standard high-resolution finite volume numerical MHD code on structured body-fitted grids. In addition, one model flow is presented which is not field-aligned, and it is discussed how grid convergence can be studied for this flow. By formal grid convergence studies of magnetic flux conservation and other flow quantities, it is investigated whether the Powell source term approach to controlling the $\nabla \cdot \boldsymbol{B}$ constraint leads to correct results for the class of flows under consideration. (c) 2001 Academic Press

Key Words: magnetohydrodynamics; shock waves; theory of characteristics; stability and convergence of numerical methods.

\section{INTRODUCTION}

In the past decade there have been many new and interesting developments in the field of numerical methods for the simulation of non-linear magnetohydrodynamic (MHD) flows with shocks $[1,4,5,8,12-14,17,31,34,36,39-41,49,50,54,55,58]$. These developments

\footnotetext{
${ }^{1}$ Research Assistant of the Fund for Scientific Research-Flanders (Belgium). Also at the High Altitude Observatory, NCAR, P.O. Box 3000, Boulder, Colorado 80307-3000, USA.

${ }^{2}$ Research Associate of the Fund for Scientific Research-Flanders (Belgium).
} 
have been driven by the increased need for robust and accurate simulation methods to tackle problems in astrophysics [29] and laboratory plasma physics [6].

Standard techniques from computational fluid dynamics (CFD) have been adapted for MHD applications. High-resolution finite-volume Godunov schemes with Riemann solvers on structured grids have been developed [1, 4, 8, 12-14, 17, 31, 34, 36, 39-41, 49, 50, 55, 58] and have been shown to perform well in a wide range of applications (e.g., [21, 29, 32, $45,48]$ ). More recently, unstructured grid codes have been presented as well [5, 11, 52].

\subsection{Quantitative Measures of Numerical Accuracy}

Thorough testing of new numerical schemes is an essential part of the development process. Most articles on new algorithmic techniques include some kind of validation in terms of model problems. However, a vast majority of the model problems presented in the MHD literature only allow very qualitative comparison and validation. Conclusions on the validity and accuracy of numerical approaches are often based solely on visual comparison of contour plots. It may be useful to determine if various numerical schemes can "capture" all the different MHD wave features properly. A new two-dimensional (2D) test problem which seems to be appropriate for this kind of comparison because it involves interacting fast and intermediate shocks and tangential discontinuities has recently been described by De Sterck et al. [45]. It is clear, however, that more quantitative measures are desirable to establish more basic formal proofs of accuracy of a numerical code, for example in terms of convergence with a certain order towards the (analytical) exact solution as a function of grid resolution ( grid convergence of the spatial discretization). The lack of model problems that allow for such an accuracy study is often attributed to the unavailability of analytical solutions to the MHD equations, especially in two or three space dimensions.

The aim of the present paper is to contribute to a remedy for the lack of model problems which allow formal study of the basic accuracy of MHD codes. We provide a set of five stationary planar $\left(B_{z} \equiv 0\right.$ and $\left.v_{z} \equiv 0\right) 2 \mathrm{D}$ field-aligned $(\boldsymbol{v} \| \boldsymbol{B})$ transonic MHD model flows which allow to study grid convergence of numerical codes. Additionally we present one model problem which is not field-aligned. We will show grid convergence for the numerical simulation of these flows with a standard high-resolution finite-volume numerical MHD code on structured body-fitted grids. Except for one of the five presented field-aligned problems, the full analytical solution is not known, but we show that grid convergence studies can be based on invariant properties following from the rigorously derived characteristic theory $[10,24,46]$ of planar stationary field-aligned MHD flow. Characteristic analysis reveals basic invariants of the flow, and these invariants can be used to test grid convergence as they lead to analytical solutions for some combinations of the flow variables if the incoming flow is uniform. Characteristic analysis also provides clear insight into the physical properties of the model flows and into the stationary wave features present in the flows. We present five field-aligned model flows, with increasing complexity in terms of wave features present in the flow: a fully smooth radial outflow, an expanding tube flow with a weak discontinuity, a wedge flow with a plane fast shock, a bow shock flow with a curved fast shock, and a nozzle flow with reflecting fast shocks. These model flows describe various nonlinear wave phenomena in their most basic form. The corresponding Euler flows have been described and used many times, but in the numerical MHD literature these basic flows have not received much attention. We will show that this set of model problems is well suited to testing grid convergence of MHD codes and to studying in detail how the behavior 
of numerical schemes changes when increasingly complex wave structures are present in the flow. Although these five model problems exhibit quite a variety of flow features, they all belong to the sub-class of stationary planar 2D field-aligned MHD flows. Stationary flow has important applications [19-21, 30, 32, 45, 51] and we show [46] that any stationary 2D problem where ideal conducting wall boundary conditions are present automatically leads to a solution where the magnetic field is aligned to the velocity field in the whole simulation domain. Hence the class of stationary field-aligned flow is an important class of MHD flows. One could even argue that 2D stationary flow problems in a finite domain with the magnetic field not aligned to the plasma flow are rare [19, 23, 30]. It is hard to define the boundary conditions consistently in that case. However, it is important to test MHD codes also for non-field-aligned flow. Therefore we include one model problem for which the magnetic field is not aligned to the plasma flow. This model flow is constructed in a special way; the fields are actually aligned in a rotating frame, but not in the rest frame. The analysis of this model problem is substantially different from the analysis of the field-aligned problems. It would certainly be useful to develop more general test problems allowing grid convergence studies, which, for instance, would also investigate the accuracy of time integration, but in this paper we take a first step and restrict ourselves to model problems belonging to the important sub-class of stationary planar flows in 2D.

Although most model problems presented in the MHD literature only allow qualitative comparison and cannot be used for formal accuracy testing, a very limited number of (mostly one-dimensional (1D)) test problems which allow some degree of formal accuracy testing have been described. Stone et al. $[49,50]$ present $1 \mathrm{D}$ model problems including stellar wind flows and obtain grid convergence which is satisfactory for most cases. Ryu et al. [41] show that the numerical dissipation of their scheme vanishes in second order as a function of grid resolution. Vanden Abeele and Deconinck [1] show how the conservation of magnetic flux in a flux tube can be used as a measure of improved accuracy as a function of grid resolution. Barth [5] obtains optimal grid convergence for the $\nabla \cdot \boldsymbol{B}$ constraint in a continuous PrandtlMeyer flow with added magnetic field. The most interesting approach to grid convergence studies of MHD codes to date can probably be found in Linde's Ph.D. thesis $[31,36]$. This author describes briefly a wedge flow and a stellar wind flow and obtains satisfactory grid convergence. Although we have made use of some of the ideas presented in the above listed earlier articles, the variety of problems presented in this paper and especially the firm grounding of convergence study on the rigorous and complete characteristic analysis of the model flows are new compared to these earlier approaches to grid convergence study of MHD codes.

\subsection{Strategies for Preservation of the $\nabla \cdot \boldsymbol{B}=0$ Condition}

The numerical enforcement of the $\nabla \cdot \boldsymbol{B}$ constraint is an important and much debated problem for numerical MHD codes. The $\nabla \cdot \boldsymbol{B}$ condition is an initial condition, which is exactly preserved in time by the partial differential equations (PDEs) of MHD, but which is not always exactly preserved after discretization of the equations (see, e.g., [36]) which may lead to numerical instability. Various strategies have been proposed to deal with the $\nabla \cdot \boldsymbol{B}$ constraint. We do not intend to give a full discussion of this subtle subject here, but we find it useful to give a brief overview of the various approaches to this problem. The equations can be formulated in terms of a vector potential, which implies divergence-free magnetic fields. This approach is not always very practical and leads to difficulties near 
sharp gradients because of the presence of second-order derivatives in the equations (see, e.g., [50]). Staggered grid approaches store different state variables on different positions of the grid, in such a way that the discrete time evolution of the magnetic field automatically conserves the divergence-free condition (e.g., [3, 14-16, 50, 55]). They often require, however, extra interpolations to be performed, and the interpolated magnetic field is not necessarily divergence-free. Subtle approaches which are intended to overcome these interpolation problems have been proposed recently $[3,14,55]$. Projection scheme approaches solve an elliptic equation in every time-step and add a computed correction to the magnetic fields to achieve divergence-free fields $[7,41,55,58]$. Solution of the elliptic equation may take considerable computing time, and spurious oscillations can also be generated in supersonic regions that should not be affected because theoretically they cannot be reached by wave perturbations in the hyperbolic system. Recently a new approach has been presented by Powell et al. [36]. These authors propose to add a source term proportional to $\nabla \cdot \boldsymbol{B}$ to the conservative form of the MHD equations. Discretization of this Galilean-invariant symmetrizable form of the equations with a source term $[5,18,36]$ leads to a stable numerical scheme. The $\nabla \cdot \boldsymbol{B}$ constraint is not enforced strongly and $\nabla \cdot \boldsymbol{B}$ can sometimes be substantially different from zero, but because of the presence of the source terms the dynamical effect of the $\nabla \cdot \boldsymbol{B}$ errors is largely neutralized and $\nabla \cdot \boldsymbol{B}$ errors can be shown to be advected away with the plasma flow. Although this approach seems to work well $[5,11,31,32,36$, $38,44,46,55]$ and has several conceptual advantages over other techniques because of its simplicity and consistency with the hyperbolic nature of the MHD equations, not much can be found in the literature about rigorous validation of this approach, and consequently it is still heavily debated. In this paper we employ the Powell source term technique and we investigate by formal grid convergence studies of magnetic flux conservation and other flow quantities whether this approach is valid, at least for the class of stationary flow problems that we consider. Linde [31] and Barth [5] carry out a similar study and confirm the validity of the Powell source term approach, but their investigation is less complete and systematic than ours and is carried out on adaptively refined Cartesian grids [31] or on unstructured grids [5], and not on the body-fitted structured grids discussed in this paper. It may be that for some problems, for which the conservation of magnetic flux up to very high accuracy is crucial, the source term technique would turn out to be insufficient. Recently Toth [55] has given an example of a time-dependent strong Riemann problem flow for which the source term approach does not seem to work satisfactorily.

\subsection{Philosophy and Organization of the Paper}

The analysis of MHD model flows in terms of stationary characteristics and the discussion of grid convergence methodology and results are the main topics treated in the present forum. We choose to make the paper self-contained and easily accessible by including brief reviews of the characteristic theory of stationary MHD and of the numerical technique we use for the simulations. We want to state clearly from the beginning that it is the philosophy of this paper to illustrate how grid convergence on the whole simulation domain-including boundaries - can be proved for numerical simulations obtained with a simple, standard, and general-purpose numerical scheme, with standard boundary conditions, limiting, interpolation, and time integration, with near-uniform grids, and with a simple and robust numerical flux function. This is an important first step and sets an initial standard of grid convergence and accuracy. Using the same criteria, it is then possible to evaluate more advanced numerical schemes in a quantitative way. In Csik et al. [11] some of the model flows and grid 
convergence criteria presented in this paper are used to evaluate a new residual distribution MHD scheme on triangular grids.

This paper is organized as follows. In Section 2 we briefly describe the MHD equations and the properties of MHD shocks. We present a compact derivation of the characteristic properties of stationary 2D planar field-aligned MHD flow. Our derivation of the characteristic properties is based on the Galilean-invariant symmetrizable form of the conservative MHD equations with a source term $[5,18,36]$ and using a matrix approach. In Section 3 we discuss the five stationary $2 \mathrm{D}$ planar field-aligned transonic model flows of increasing complexity. We discuss the flow features present in each flow in terms of stationary characteristics. A non-field-aligned model problem is discussed in Section 4. In Section 5 we give a brief but complete discussion of the standard high-resolution finite-volume numerical MHD scheme on structured body-fitted grids. Next we formulate practical grid convergence criteria based on the invariants revealed by the characteristic analysis. In Section 6 we discuss the grid convergence results for simulation of the model flows and their implication for the validity of the Powell source term approach. We formulate our conclusions in Section 7.

\section{THE MHD EQUATIONS, MHD DISCONTINUITIES, AND THE THEORY OF STATIONARY CHARACTERISTICS}

\subsection{The MHD Equations}

The equations of ideal one-fluid MHD in conservative form [36] are given by

$$
\frac{\partial}{\partial t}\left[\begin{array}{c}
\rho \\
\rho v \\
\boldsymbol{B} \\
e
\end{array}\right]+\nabla \cdot\left[\begin{array}{c}
\rho \boldsymbol{v} \\
\rho \boldsymbol{v} \boldsymbol{v}+I(p+\boldsymbol{B} \cdot \boldsymbol{B} / 2)-\boldsymbol{B} \boldsymbol{B} \\
\boldsymbol{B} \boldsymbol{v}-\boldsymbol{v} \boldsymbol{B} \\
(e+p+\boldsymbol{B} \cdot \boldsymbol{B} / 2) \boldsymbol{v}-(\boldsymbol{v} \cdot \boldsymbol{B}) \boldsymbol{B}
\end{array}\right]=-\left[\begin{array}{c}
0 \\
\boldsymbol{B} \\
\boldsymbol{v} \\
\boldsymbol{v} \cdot \boldsymbol{B}
\end{array}\right] \nabla \cdot \boldsymbol{B}
$$

This equation has to be supplemented with the divergence-free condition $\nabla \cdot \boldsymbol{B}=0$ as an initial condition. Here, $\rho$ and $p$ are the plasma density and pressure, respectively, $\boldsymbol{v}$ is the plasma velocity, $\boldsymbol{B}$ is the magnetic field, and

$$
e=\frac{p}{\gamma-1}+\rho \frac{\boldsymbol{v} \cdot \boldsymbol{v}}{2}+\frac{\boldsymbol{B} \cdot \boldsymbol{B}}{2}
$$

is the total energy density of the plasma. $I$ is the unity matrix. The magnetic permeability $\mu=1$ in our units. We take $\gamma=5 / 3$ for the adiabatic index. These equations describe the conservation of mass, momentum, magnetic field, and energy. For simplicity, we consider a perfect gas, such that the sound speed is given by

$$
c=\sqrt{\gamma p / \rho}
$$

and the entropy is given by

$$
s=p / \rho^{\gamma} .
$$

The hydrodynamic stagnation enthalpy is defined as

$$
h_{s} \equiv \frac{\gamma}{\gamma-1} \frac{p}{\rho}+\frac{1}{2} v^{2}
$$

and plays a role in the characteristic analysis of field-aligned MHD flow. 
We have written Eq. (1) in a form with a source term proportional to $\nabla \cdot \boldsymbol{B}$ on the right hand side (RHS). This form of the equations, which reduces to the more familiar physical conservation laws when we set $\nabla \cdot \boldsymbol{B}=0$, can be useful for certain purposes. Godunov $[5,18]$ proved in 1972 that this is the unique form of the MHD equations which is symmetrizable when $\nabla \cdot \boldsymbol{B}$ is non-vanishing. It is a more general form of the MHD equations, in the sense that this form of the equations remains Galilean-invariant also if $\nabla \cdot \boldsymbol{B} \neq 0$ [36]. Discretization of this form of the equations is one way to control the $\nabla \cdot \boldsymbol{B}$ constraint in conservative, shock-capturing numerical schemes [36].

The inclusion of this RHS term is also essential for the derivation of the characteristic theory of stationary 2D MHD in a simple, compact, and systematic procedure using a matrix approach, as is described in [46], and in Section 2.3 for the special case of planar field-aligned flow.

\subsection{Discontinuities and Rankine-Hugoniot Relations}

Contrary to the hydrodynamic equations, which allow for only one wave mode, the MHD equations allow for three distinct wave modes, the fast magneto-acoustic wave, the Alfvén wave, and the slow magneto-acoustic wave, with (positive) anisotropic wave speeds satisfying $c_{\mathrm{f}} \geq c_{\mathrm{A}} \geq c_{\mathrm{s}}$ in standard notation. The ideal MHD equations allow for discontinuous solutions, and those discontinuities have to satisfy the MHD Rankine-Hugoniot (RH) relations [27], which in the shock frame read

$$
F\left(\mathbf{U}_{l}\right)=F\left(\mathbf{U}_{r}\right)
$$

with $\mathbf{U}_{l}$ and $\mathbf{U}_{r}$ the left and right state vectors of conserved variables, and $F$ the 1D flux function which follows from Eq. (1). The MHD flux function (in the $x$ direction) is given by

$$
F\left(\left[\begin{array}{c}
\rho \\
\rho v_{x} \\
\rho v_{y} \\
\rho v_{z} \\
B_{x} \\
B_{y} \\
B_{z} \\
e
\end{array}\right]\right)=\left[\begin{array}{c}
\rho v_{x} \\
\rho v_{x}^{2}+p+B^{2} / 2-B_{x}^{2} \\
\rho v_{x} v_{y}-B_{x} B_{y} \\
\rho v_{x} v_{z}-B_{x} B_{z} \\
0 \\
B_{y} v_{x}-B_{x} v_{y} \\
B_{z} v_{x}-B_{x} v_{z} \\
\left(e+p+B^{2} / 2\right) v_{x}-B_{x}(\boldsymbol{v} \cdot \boldsymbol{B})
\end{array}\right] .
$$

The RH relations basically describe the continuity of the mass, momentum, magnetic field, and energy fluxes through the discontinuity. Three types of shocks are described by the MHD RH relations, connecting plasma states which are traditionally labeled from 1 to 4 , with state 1 a super-fast state; state 2 sub-fast but super-Alfvénic; state 3, sub-Alfvénic but super-slow; and state 4, sub-slow [2, 27, 45]. Fast 1-2 MHD shocks refract the magnetic field away from the shock normal, intermediate MHD shocks (1-3, 1-4, 2-3, and $2-4)$ change the sign of the component of the magnetic field which is tangential to the shock front and thus flip magnetic field lines over the shock normal, and slow 3-4 MHD shocks refract the magnetic field towards the shock normal. For shocks, there is both a mass flow through the surface of discontinuity, and an increase in the entropy $s$. Contact 
discontinuities, with vanishing $v_{x}$, but nonzero $B_{x}$, have only a jump in density (and entropy). All other quantities are continuous. Tangential discontinuities, with vanishing $v_{x}$ and $B_{x}$, have a jump in density, pressure, and tangential velocity and field. However, the total pressure $p+B^{2} / 2$ is continuous. Rotational discontinuities rotate the magnetic field around the normal of the discontinuity surface over an arbitrary angle, without a jump in entropy.

There is general agreement that fast and slow shocks can exist physically, but the subject of the physical admissibility of intermediate MHD shocks and rotational discontinuities has a long history and is still very much debated. See [34, 45] for an introduction and references. Recently intermediate shocks have been identified in stationary 2D and 3D MHD bow shock flows $[45,47]$.

\subsection{Characteristic Analysis of Stationary Field-Aligned MHD Flow}

Results on characteristic theory of the MHD equations can be found scattered throughout the literature $[10,24]$. We have chosen, however, to make this paper self-contained by providing a compact derivation of the characteristic theory for the sub-class of stationary 2D planar field-aligned MHD flows [22, 24, 25]. This brief derivation serves to introduce the reader who is unfamiliar with the theory of characteristics to the concepts, nomenclature, and notation that is used further on in the paper. Our derivation of the characteristic properties is based on the Galilean-invariant symmetrizable form of the conservative MHD equations with a source term $[5,18,36]$ and using a matrix approach [24]. A more complete derivation of the characteristic properties of MHD using this compact matrix approach (not restricted to the special case of stationary planar field-aligned flow), can be found in De Sterck et al. [46].

First we prove that if the magnetic field and the velocity field are aligned at one point of a stationary planar 2D MHD flow, then the magnetic field is aligned to the velocity field everywhere. The classical form of the induction equation reads $\partial \boldsymbol{B} / \partial t=-\nabla \times \boldsymbol{E}$. Thus $\partial \boldsymbol{B} / \partial t=-\nabla \times \boldsymbol{E}=0$ leads to $\partial E_{z} / \partial x=\partial E_{z} / \partial y=0$. This means that $E_{z}$, which is given by $E_{z}=-\boldsymbol{v} \times \boldsymbol{B}$ in planar ideal MHD, is a constant over the whole $x y$ plane. This is also true when discontinuities are present in the flow, as the tangential component of the electric field is continuous at discontinuities [27]. If $\boldsymbol{v} \| \boldsymbol{B}$ in some point, which implies that $E_{z}=0$ there, then $E_{z}=0$ everywhere, which implies that the magnetic field is aligned to the velocity field everywhere. This property is a consequence of the fact that the magnetic field is frozen into the plasma flow for an ideal MHD plasma [27]. For instance, if a stationary 2D flow problem contains a perfectly conducting wall (where the magnetic field and the velocity field have to be aligned to the wall), then the magnetic field will be aligned to the velocity field at every point of the flow. The concept of stationary field-aligned flow is thus well defined and establishes an important class of stationary MHD flows [19, 46].

We rewrite Eq. (1) with $\partial / \partial t \equiv 0, B_{z} \equiv 0$, and $v_{z} \equiv 0$ (planar flow) in terms of the primitive variables $\mathbf{W}=\left(\rho, v_{x}, v_{y}, B_{x}, B_{y}, p\right)$ and introduce the variable $\alpha$ by taking $\boldsymbol{B}=$ $\alpha \boldsymbol{v}$. The steady MHD equations for planar field-aligned flow then reduce to a $5 \times 5$ system which can be written in quasi-linear form,

$$
\mathbf{A}(\mathbf{W}) \cdot \frac{\partial \mathbf{W}}{\partial x}+\mathbf{B}(\mathbf{W}) \cdot \frac{\partial \mathbf{W}}{\partial y}=0
$$


with $\mathbf{W}=\left(\rho, v_{x}, v_{y}, \alpha, p\right)$, and matrices

$$
\mathbf{A}=\left[\begin{array}{ccccc}
v_{x} & \rho & 0 & 0 & 0 \\
0 & v_{x} & \alpha^{2} v_{y} / \rho & \alpha v_{y}^{2} / \rho & 1 / \rho \\
0 & 0 & \left(1-\alpha^{2} / \rho\right) v_{x} & -\alpha v_{x} v_{y} / \rho & 0 \\
0 & \alpha & 0 & v_{x} & 0 \\
0 & c^{2} \rho & 0 & 0 & v_{x}
\end{array}\right]
$$

and

$$
\mathbf{B}=\left[\begin{array}{ccccc}
v_{y} & 0 & \rho & 0 & 0 \\
0 & \left(1-\alpha^{2} / \rho\right) v_{y} & 0 & -\alpha v_{x} v_{y} / \rho & 0 \\
0 & \alpha^{2} v_{x} / \rho & v_{y} & \alpha v_{x}^{2} / \rho & 1 / \rho \\
0 & 0 & \alpha & v_{y} & 0 \\
0 & 0 & c^{2} \rho & 0 & v_{y}
\end{array}\right] .
$$

The characteristic properties of this system of equations can be derived by analyzing the equations in the form

$$
\frac{\partial \mathbf{W}}{\partial x}+\mathbf{A}^{-1} \cdot \mathbf{B} \cdot \frac{\partial \mathbf{W}}{\partial y}=0 .
$$

The eigenvalues of the matrix $\mathbf{C} \equiv \mathbf{A}^{-1} \cdot \mathbf{B}$ determine the type (hyperbolic or elliptic) of the five separate characteristic fields of the system. Hyperbolicity depends on the value of the state variable $\mathbf{W}$. A characteristic field is hyperbolic if its associated eigenvalue is real and thus defines a real characteristic direction in the $x y$ plane. For those real characteristic fields, Riemann invariants (RIs) may exist which describe quantities which are conserved on their associated characteristics. Those RIs can be found by analysis of the left eigenvectors of matrix C. When a characteristic field is elliptic complex eigenvalues are obtained. More information on these concepts and this methodology can be found in [9, 10, 46].

For purposes of comparison, we repeat here the basic results for the characteristic properties of stationary planar hydrodynamic (Euler) flow, with which the reader may be familiar $[9,10]$. In this case the state vector is given by $\mathbf{W}=\left(\rho, v_{x}, v_{y}, p\right)$, and there are four characteristic fields. The streamlines are twofold-degenerate characteristics and the entropy and the stagnation enthalpy are the associated RIs. The equations are fully hyperbolic when the flow is supersonic, and in supersonic regions the Mach lines are characteristics of the equations which exist in real space, making equal angles (Mach angles) with the streamlines. For subsonic flow, the Mach lines do not exist and the associated characteristic fields are elliptic.

Analysis of Eq. (11) leads to similar results for the characteristic properties of stationary field-aligned MHD flow.

The characteristic condition $\operatorname{det}(\mathbf{C}-\lambda \mathbf{I})=0$ can be factored in terms of the variable

$$
v_{\perp}^{2}=\frac{\left(-v_{y}+v_{x} \lambda\right)^{2}}{1+\lambda^{2}},
$$


which represents the square of the velocity component perpendicular to the characteristic, as the direction of the characteristic is given by $d y / d x=\lambda$. This factorization leads to the roots

$$
v_{\perp 1}^{2}=c^{2}\left(1-\alpha^{2} / \rho\right)+v^{2} \alpha^{2} / \rho \quad \text { and } \quad v_{\perp 2}^{3}=0 .
$$

The equation for $v_{\perp 2}$ always has three real solutions, $\lambda_{3,4,5}=v_{y} / v_{x}$, meaning that the streamlines are threefold degenerate characteristics. The corresponding left eigenvectors are

$$
\begin{gathered}
\mathbf{L}_{3}=\left(-c^{2}, 0,0,0,1\right), \quad \mathbf{L}_{4}=(-\alpha / \rho, 0,0,1,0), \quad \text { and } \\
\mathbf{L}_{5}=\left(c^{2} /\left(\rho v_{y}\right), v_{x} / v_{y}, 1,0,0\right),
\end{gathered}
$$

and can be used to derive the Riemann invariants

$$
\chi_{3}=s, \quad \chi_{4}=\rho / \alpha, \text { and } \chi_{5}=h_{s} .
$$

For instance, the condition that $\mathbf{L}_{3} \cdot d \mathbf{W}=0$ in the direction of the characteristic with slope $v_{y} / v_{x}$ can be written as $\mathbf{L}_{3} \cdot\left(d \rho, d v_{x}, d v_{y}, d \alpha, d p\right)=-c^{2} d \rho+d p=0$. This leads to $d s=0$, because it follows from Eq. (4) that $d s=d p / \rho^{\gamma}-\gamma p / \rho^{\gamma+1} d \rho=\left(-c^{2} d \rho+\right.$ $d p) / \rho^{\gamma}$. This means that the entropy $s$ is a RI. The entropy, $\rho / \alpha$, and the stagnation enthalpy are thus conserved on a streamline in continuous flow. It can be derived from the MHD RH jump conditions (6) that the entropy is discontinuous when a streamline crosses a shock. However, the stagnation enthalpy and $\rho / \alpha$ are conserved over a shock for field-aligned flow.

The solutions of the equation for $v_{\perp 1}$ are

$$
\lambda_{1,2}=\frac{\rho v_{x} v_{y} \pm \sqrt{\left(v^{2}-c^{2}\right)\left(\alpha^{2}-\rho\right)\left(c^{2}\left(\alpha^{2}-\rho\right)-v^{2} \alpha^{2}\right)}}{\rho\left(c^{2}-v_{x}^{2}\right)-\alpha^{2}\left(c^{2}-v^{2}\right)} .
$$

If the factor under the square root sign is positive, then these eigenvalues are real, and the equations are hyperbolic [19, 22, 24, 25, 46]. This factor changes sign three times, viz., when the square of the velocity equals

$$
v^{2}=c_{A}^{2}, \quad v^{2}=c^{2}, \quad \text { and } \quad v^{2}=c_{\text {cusp }}^{2} .
$$

The cusp velocity is defined as $c_{\text {cusp }}^{2}=\left(c^{2} c_{\mathrm{A}}^{2}\right) /\left(c^{2}+c_{\mathrm{A}}^{2}\right)$ and is the velocity of the slow wave cusp in the MHD Friedrichs diagram [22,24]. Note that we define $c_{\mathrm{A}}$ as the Alfvén speed in the direction of the magnetic field in the case of field-aligned flow. This leads to a division of the parameter space into elliptic and hyperbolic regions, as depicted in Fig. 1, for high

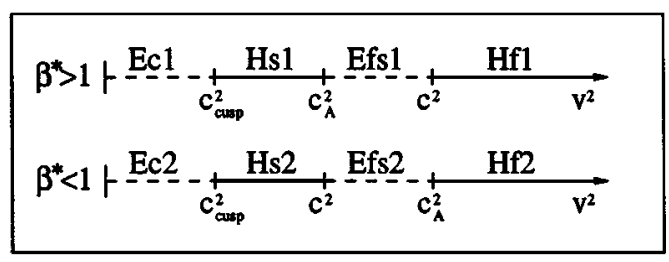

FIG. 1. Elliptic and hyperbolic regions in parameter space for steady planar field-aligned MHD. The top line corresponds to $\beta^{*}>1$ and shows, as $v^{2}$ decreases, a division in a fast hyperbolic region (Hf1), an elliptic region between the fast and the slow hyperbolic regions (Efs1), a slow hyperbolic region (Hs1), and an elliptic region below the cusp speed (Ec1). The bottom line shows a similar division for $\beta^{*}<1$. For $\beta^{*}=1, c^{2}=c_{A}^{2}$. 
and low $\beta^{*}$, where the parameter $\beta^{*}$ is defined as $\beta^{*}=p /\left(B^{2} / \gamma\right)=c^{2} / c_{\mathrm{A}}^{2}=M_{\mathrm{A}}^{2} / M^{2}$, with the sonic and Alfvénic Mach numbers defined as $M=v / c$ and $M_{\mathrm{A}}=v / c_{\mathrm{A}}$.

The local geometry of streamlines and $x y$ characteristics for the case of planar steady field-aligned MHD in hyperbolic regions is very similar to the Euler case. The streamline is a threefold degenerate characteristic, and there are two additional families of characteristics (generalized Mach lines $[10,9]$ ) which make equal angles $\psi$ with the streamline. These characteristics can be of the slow or fast type, depending on which hyperbolic regime the parameters are in (Hf or Hs of Fig. 1).

We are now ready to use this characteristic theory for the analysis of the physical properties of five 2D planar field-aligned MHD model flows of increasing complexity in the next section. In Section 5.6 we use this characteristic theory for the formulation of grid convergence criteria for 2D MHD flows belonging to the class of planar field-aligned flows. In Section 6 these criteria are used to study the grid convergence of the numerical simulation of the five field-aligned model flows with our MHD scheme.

\section{PHYSICAL ANALYSIS OF FIELD-ALIGNED MODEL FLOWS IN TERMS OF STATIONARY CHARACTERISTICS}

In this section we present five model flows of increasing complexity belonging to the class of stationary planar field-aligned MHD flow. We analyze the flows and the stationary wave structures present in terms of stationary characteristics. The figures shown in this section are actual simulation results obtained with our numerical MHD code using a second-order scheme, but we defer discussion of the numerical aspects of the simulations to Sections 5 and 6.

\subsection{Cylindrical Expansion Flow}

The first model flow (Fig. 2) is a stationary cylindrical expansion flow in the domain $\left(r \in[1,2], \theta \in\left[0^{\circ}, 30^{\circ}\right]\right)$. A uniform superfast radial inflow with radial magnetic field is

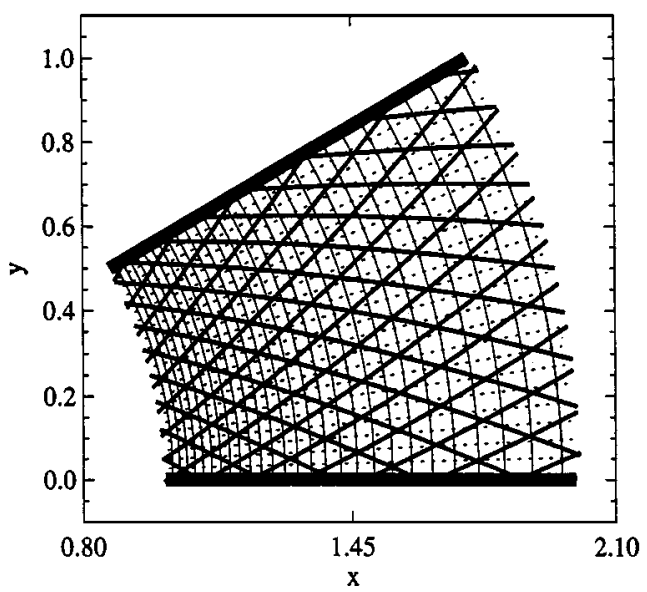

FIG. 2. The cylindrical expansion flow $(70 \times 70$ grid). Density contours (thin solid) and streamlines (thin dotted) are shown. The streamlines are also magnetic field lines. The thick solid lines represent simulation domain boundaries with ideal wall symmetry. The flow is hyperbolic in the whole simulation domain. Two families of fast characteristics (thick solid) make equal angles with the streamlines. The flow is smooth in the whole simulation domain. 
imposed at the $r=1$ boundary, with $\rho=1, p=1, v_{r}=3$, and $B_{r}=1$. The sonic and Alfvénic inflow Mach numbers are thus $M=2 \sqrt{3} / \sqrt{5}$ and $M_{\mathrm{A}}=3$. The plasma is allowed to flow out freely at $r=2$. For $r>1$, a stationary completely smooth radial expansion profile with variables only changing as a function of $r$ results. This flow is thus strictly speaking a $1 \mathrm{D}$ flow.

In Fig. 2 and the subsequent figures, density contours are shown as thin solid lines, and streamlines as thin dotted lines. The streamlines are also magnetic field lines. The streamlines are threefold-degenerate characteristics with $s, h_{s}$, and $\rho / \alpha$ as associated RIs. The thick solid lines represent simulation domain boundaries with ideal wall symmetry. Two families of fast characteristics (thick solid) make equal angles with the streamlines in hyperbolic regions.

As the inflow in Fig. 2 is uniform and every streamline thus carries the same values for the RIs $s, h_{s}$, and $\rho / \alpha$, those RIs are global invariants over the whole flow domain. $\nabla \cdot \boldsymbol{B}=0$ and $B_{r}(1)=1$ imply that $B_{r}(r)=1 / r$. The four state variables $\rho, p, B_{r}$, and $v_{r}$ can thus be determined as functions of $r$ from $B_{r}(r)=1 / r$ and the three global invariants, which establishes the exact analytical solution of this flow problem. The flow is hyperbolic Hf1 (Fig. 1) everywhere, so the two (generalized) Mach characteristics exist. This flow is smooth everywhere, and this is the property which distinguishes it from the model flows to be presented next, which contain increasingly complex (weakly) discontinuous flow features. This flow is related to the stellar outflow problems discussed in $[26,31,42,49$, $50]$. By giving the flow a rotational component at the inflow boundary, we obtain a nonfield-aligned model problem describing radial outflow from a rotating object [26, 42]. This rotating outflow problem is described and analyzed in Section 4.

\subsection{Expanding Tube Flow}

In Fig. 3 we shows a stationary expanding tube flow in the domain $(x \in[0,1], y \in$ $\left.\left[y_{0}(x), 1\right]\right)$, with $y_{0}(x)=0$ for $x \in[0,0.3]$ and $y_{0}(x)=-1+\cos (\pi / 4 *(x-0.3))$ for $x \in[0.3,1]$. A uniform superfast horizontal inflow with horizontal magnetic field is imposed at the $x=0$ boundary, with $\rho=1, p=1, v_{x}=8$, and $B_{x}=4$. The sonic and Alfvénic inflow Mach numbers are thus $M=8 \sqrt{3} / \sqrt{5}$ and $M_{\mathrm{A}}=2$. The plasma is allowed to flow out freely at $x=1$, where the flow remains superfast. At $y=y_{0}(x)$ and $y=1$ we impose ideal wall symmetry conditions. A stationary expanding flow results, as shown by the density contours of Fig. 3.

The flow is hyperbolic Hf2 (Fig. 1) in the whole simulation domain, such that two families of fast characteristics exist. As long as the lower wall is straight, the flow is uniform. When the wall starts to curve $(x=0.3)$, this acts as a (wavelike) perturbation, which can only propagate downstream along the characteristics. This means that the flow is non-uniform only below the upward fast characteristic which originates from the lower boundary at ( $x=0.3$ ) with an angle of $\theta=31.276^{\circ}$, as can be calculated from Eq. (16). This fast characteristic thus separates a uniform flow region from a perturbed region, and is thus necessarily a weak discontinuity $[9,10]$. At a weak discontinuity, the spatial derivative of the flow variables in a direction perpendicular to the weak discontinuity characteristic is discontinuous. However, the flow variables themselves are continuous, and the entropy is thus also conserved on streamlines across weak discontinuities. A weak discontinuity thus detaches from the lower wall where the tube starts to expand. This weak discontinuity is a fast characteristic, and is followed by a simple wave $[9,10,46]$ rarefaction region. 


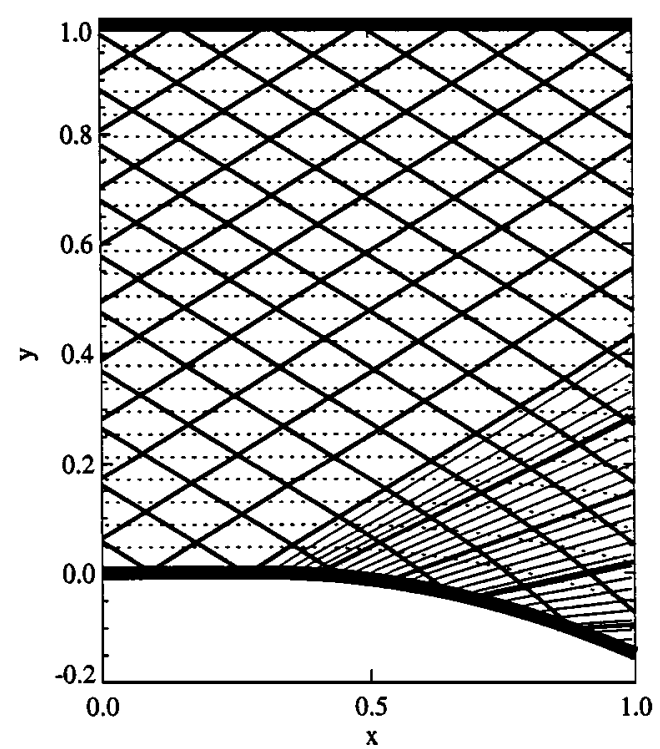

FIG. 3. The expanding tube flow $(75 \times 125$ grid $)$. The flow is hyperbolic in the whole simulation domain. A weak discontinuity detaches from the lower wall where the tube starts to expand. This weak discontinuity is a fast characteristic and is followed by a simple wave rarefaction region. One family of characteristics consists of straight lines in the simple wave region. The simple wave is not centered.

This rarefaction is called a (stationary) simple wave because it carries a variation in only one MHD wave family. It is a property of simple waves that one family of characteristics consists of straight lines and that the flow variables are constant along these characteristics, so that the characteristics are parallel to the contour lines of flow variables, e.g., the density, as can be seen in Fig. 3. In the present flow, these straight line characteristics do not converge at one point, so this simple wave is not centered. Centered simple waves exist in rarefaction flows around sharp corners, like the well-known Prandtl-Meyer flow [5]. Such a sharp corner is a geometrical singularity in the boundary and this can complicate grid convergence studies, as is explained in the next section. For this reason, we have chosen to present model flows with smooth boundaries in this paper. A simple wave, also if it is non-centered, can be described mathematically as a function of only one spatial parameter, so strictly speaking this flow is still 1D. As the inflow is uniform, every streamline throughout the whole domain carries the same values for RIs $h_{s}$ and $\rho / \alpha$, and also for $s$, as the flow does not contain strong discontinuities. These RIs are thus global invariants over the whole flow domain.

This flow contains a weak discontinuity, and this is the property which distinguishes it from the fully smooth model flow discussed in Section 3.1.

\subsection{Wedge Flow}

Figure 4 shows a stationary wedge flow in the domain $\left(x \in[0,1], y \in\left[y_{0}(x), 1.5\right]\right)$. The lower simulation domain boundary has the form of a wedge with angle $\theta=30^{\circ}$.

A uniform superfast horizontal inflow with horizontal magnetic field is imposed at the $x=0$ boundary, with $\rho=1, p=1, v_{x}=8$, and $B_{x}=4$. The sonic and Alfvénic inflow Mach numbers are thus $M=8 \sqrt{3} / \sqrt{5}$ and $M_{\mathrm{A}}=2$. At $y=y_{0}(x)$ and $y=1$ we impose ideal wall symmetry conditions. The wedge geometry causes the formation of a fast MHD 


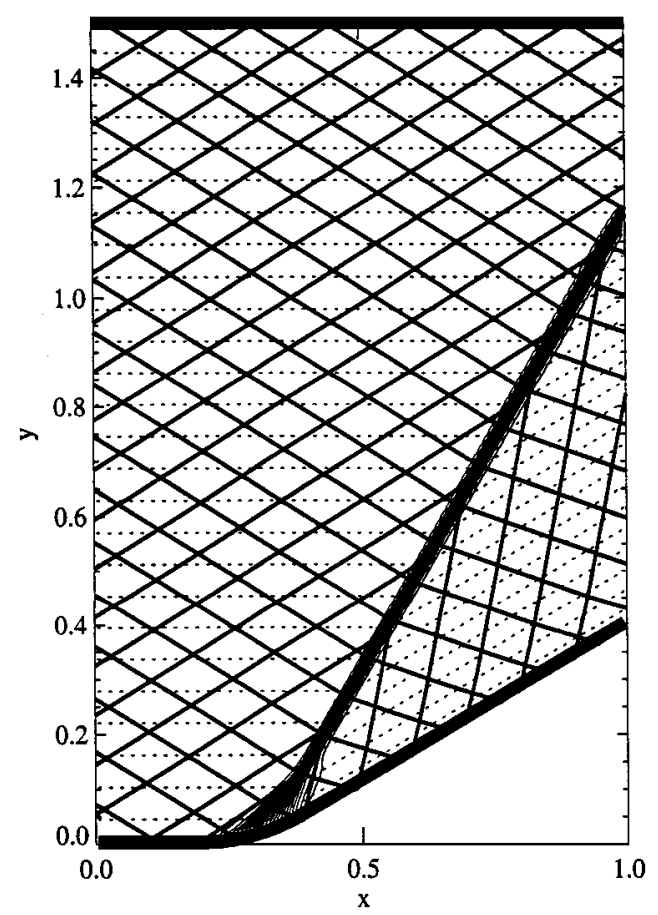

FIG. 4. The wedge flow $(100 \times 200$ grid $)$. The flow is hyperbolic in the whole simulation domain. A plane fast MHD shock is formed where characteristics start to intersect near the lower wall. This discontinuity stretches out upwards in a straight line.

shock, as shown by the accumulation of density contours in a solid line. At the shock, the magnetic field lines are refracted away from the shock normal in going from upstream to downstream, which shows that this is a fast MHD shock. The plasma is allowed to flow out freely at $x=1$, where the (normal) flow is superfast.

As the inflow is uniform, every streamline throughout the whole domain carries the same values for $h_{s}$ and $\rho / \alpha$, but not for $s$, as the flow does contain a strong discontinuity where the entropy increases discontinuously. Only the RIs $h_{s}$ and $\rho / \alpha$ are thus global invariants over the whole flow domain. The flow is hyperbolic Hf2 (Fig. 1) everywhere, so the two (generalized) Mach characteristics exist.

We have to remark that the two straight "legs" of the wedge could be connected at $x=0.3$, but that we have again chosen a smooth boundary with the wedge corner smoothed out by a circular profile. The lower boundary is described by $y_{0}(x)=0$ for $x \in[0,0.2]$ and $y_{0}(x)=$ $\tan \left(30^{\circ}\right) *(x-0.3)$ for $x \in\left[0.3+0.1 * \cos \left(30^{\circ}\right), 1\right]$. A segment of a circle with center point $\left(0.2,0.1 *\left(1+\cos \left(30^{\circ}\right)\right) / \sin \left(30^{\circ}\right)\right)$ and radius $r=0.1 *\left(1+\cos \left(30^{\circ}\right)\right) \sin \left(30^{\circ}\right)$, which is tangent to the two "legs" of the wedge, then replaces the corner singularity of the wedge with a smooth profile. Close to the lower boundary our wedge flow with smooth boundaries is thus slightly different from a wedge flow with a sharp corner, and one can see a small compression wave region with converging characteristics in the corner region on Fig. 4 [53], but above the point where those characteristics converge and the shock is formed, this flow is identical to the wedge flow with a sharp corner.

The wedge flow described in this section contains a plane strong discontinuity, and this is the property which distinguishes it from the flows discussed earlier. MHD wedge flows have also been discussed in [31]. 


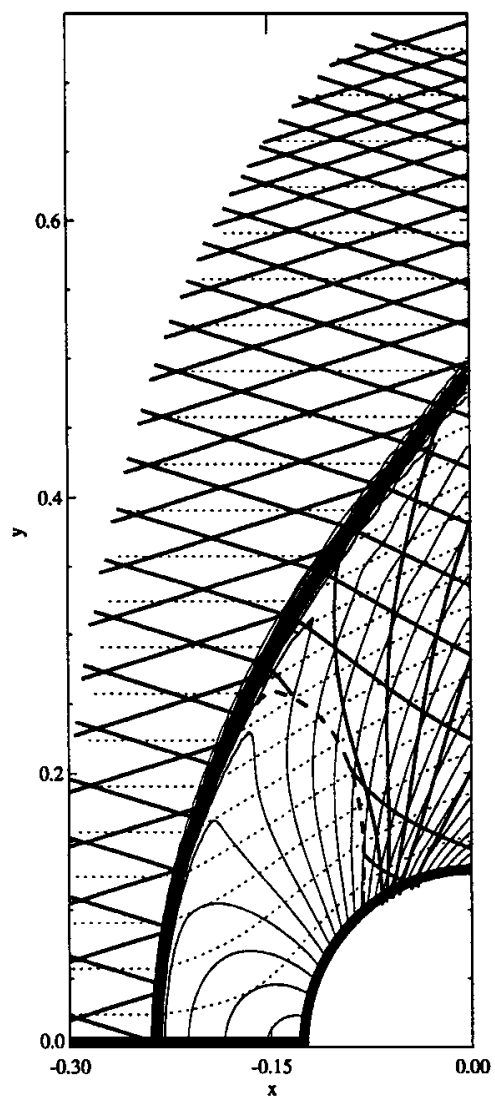

FIG. 5. The bow shock flow $(80 \times 80$ grid $)$. A fast MHD bow shock is formed in front of the cylindrical obstacle. The flow is hyperbolic in front of this bow shock, and also behind the shock front sufficiently far upward from the horizontal $x$-axis. The region behind the shock front and close to the horizontal $x$-axis is an elliptic region, in which real fast characteristics do not exist. This elliptic region is separated from the upward hyperbolic region by the $M=1$ contour (dashed).

\subsection{Bow Shock Flow}

In Fig. 5 we show a stationary bow shock flow in the domain $\left(r \in\left[r_{0}(\theta), 0.125\right], \theta \in\right.$ $\left.\left[90^{\circ}, 180^{\circ}\right]\right)$, with $r_{0}(\theta)=0.75-0.45(\theta-90) / 90$. The bow shock is formed by the obstruction of a uniform incoming flow by a rigid perfectly conducting circular cylinder with $r=0.125$. A uniform superfast horizontal inflow with horizontal magnetic field is imposed at the $r=r_{0}$ boundary with $\rho=1, p=0.2, v_{x}=2$, and $B_{x}=0.1$. The sonic and Alfvénic inflow Mach numbers are thus $M=2 \sqrt{3}$ and $M_{A}=20$. At $y=0$ and $r=0.125$ we impose ideal wall symmetry conditions. The plasma is allowed to flow out freely at $x=0$, where the (normal) flow is superfast. A curved fast MHD bow shock is formed, as shown by the accumulation of density contours. At the shock, the magnetic field lines are refracted away from the shock normal in going from upstream to downstream, which shows that this is a fast MHD shock.

As the inflow is uniform, every streamline throughout the whole domain carries the same values for $h_{s}$ and $\rho / \alpha$, but not for $s$ as the flow does contain a strong discontinuity. Only the RIs $h_{s}$ and $\rho / \alpha$ are thus global invariants over the whole flow domain. The flow is hyperbolic Hf1 (Fig. 1) in the upstream region and in the part of the downstream region above the dashed line $\left(v^{2}=c^{2}\right.$ or $M=1$ contour). In these hyperbolic regions the two 


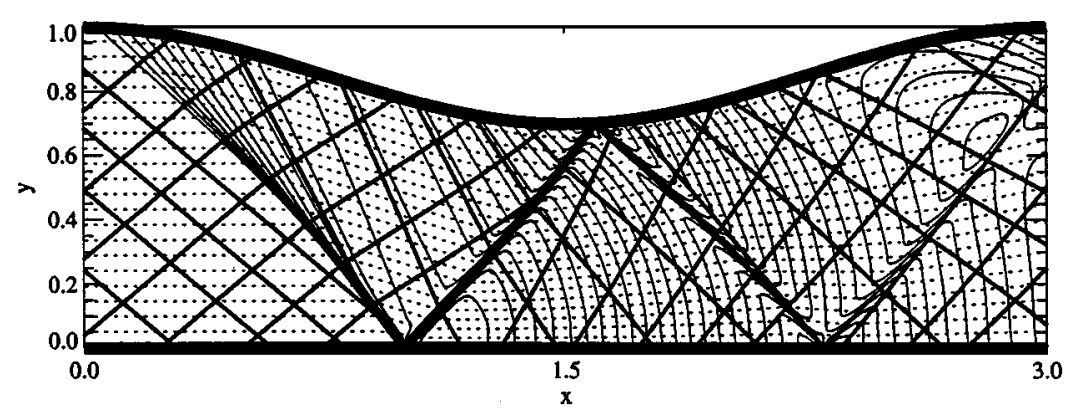

FIG. 6. The nozzle flow $(480 \times 160$ grid $)$. The flow is hyperbolic in the whole simulation domain. A fast MHD shock is formed where characteristics start to intersect. This shock is reflected by the lower wall, by the upper wall, and again by the lower wall before it leaves the simulation domain. Throughout these reflections, the shock front remains nearly plane and the shock remains of the fast type.

(generalized) Mach characteristics exist. The downstream region below the $M=1$ contour is of elliptic type Efs1, and real characteristics do not exist in this region.

The bow shock flow described in this section contains a curved strong discontinuity and an elliptic region, and these properties distinguish it from the flows discussed earlier. MHD bow shock flows have been discussed before [1, 5, 45, 46, 54]. De Sterck et al. [46] describe the characteristic analysis of a complex MHD bow shock flow which contains interacting fast and intermediate shocks and tangential discontinuities and several alternating regions of the different hyperbolic and elliptic types of Fig. 1.

\subsection{Nozzle Flow}

In Fig. 6 we show a stationary nozzle flow in the domain $\left(x \in[0,3], y \in\left[0, y_{1}(x)\right]\right)$, with $y_{1}(x)=1-0.3 * \sin ^{2}(\pi / 3 * x)$. A uniform superfast horizontal inflow with horizontal magnetic field is imposed at the $x=0$ boundary with $\rho=1, p=1, v_{x}=3.5$, and $B_{x}=2$. The sonic and Alfvénic inflow Mach numbers are thus $M=3.5 \sqrt{3} / \sqrt{5}$ and $M_{\mathrm{A}}=1.75$. At $y=0$ and $y=y_{1}$ we impose ideal wall symmetry conditions. The plasma is allowed to flow out freely at $x=3$, where the (normal) flow is superfast. A fast MHD shock is formed near the upper wall because of the curvature of this wall, as shown by the accumulation of density contours. At the shock, the magnetic field lines are refracted away from the shock normal in going from upstream to downstream, which shows that this is a fast MHD shock. This shock reflects several times from the rigid ideal walls $y=0$ and $y=y_{1}(x)$.

As the inflow is uniform, every streamline throughout the whole domain carries the same values for $h_{s}$ and $\rho / \alpha$, but not for $s$ as the flow does contain strong discontinuities. Only the RIs $h_{s}$ and $\rho / \alpha$ are thus global invariants over the whole flow domain. The flow is hyperbolic Hf2 (Fig. 1) everywhere, and two (generalized) Mach characteristics exist.

The nozzle flow described in this section contains strong discontinuities which are reflected by ideal walls, which distinguishes it from the flows discussed earlier. MHD nozzle flows have been discussed before in [1].

\section{PHYSICAL ANALYSIS OF A ROTATING OUTFLOW WITH THE FIELD NOT ALIGNED TO THE FLOW}

In this section we describe and analyze a model problem describing radial outflow from a rotating cylindrical object. We start from the cylindrical expansion flow described in 


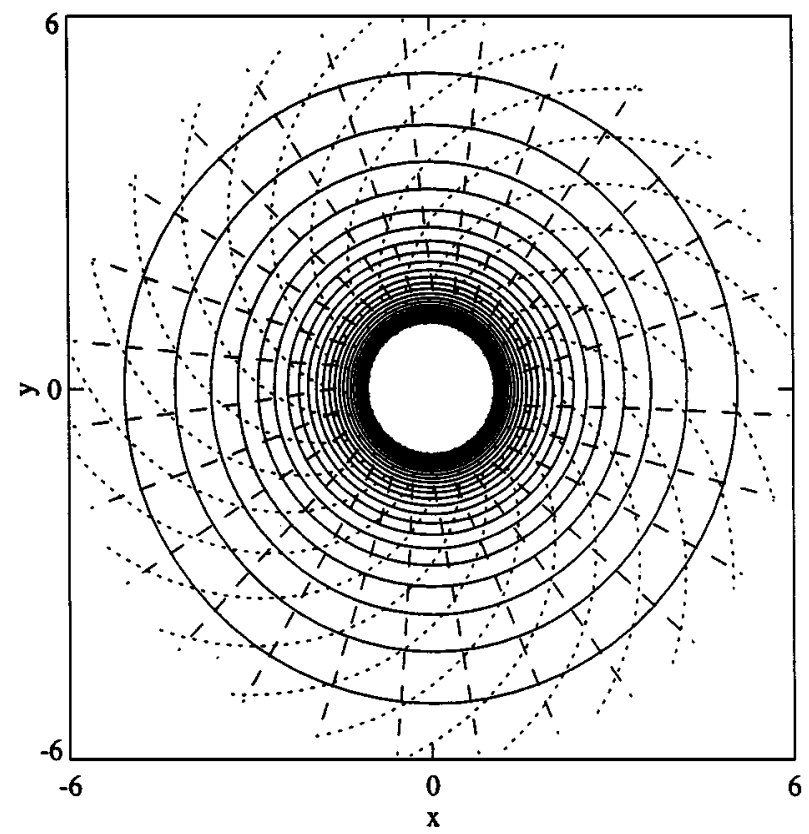

FIG. 7. The rotating outflow problem $(70 \times 70$ grid). Density contours (thin solid), streamlines (dashed), and magnetic field lines (dotted) are shown. The magnetic and velocity fields are not aligned. The flow is smooth in the whole simulation domain.

Section 3.1. We take the same inflow conditions and keep $\gamma=5 / 3$, but add a rotational velocity component $v_{\theta}=1$ in the counter-clockwise direction. The magnetic field remains radial at the boundary $r=1$. We extend the simulation domain up to $r=6$ and $\theta=360^{\circ}$. The resulting smooth stationary flow is show in Fig. 7. The magnetic field lines (dotted) are clearly not aligned with the streamlines (dashed). If we transform to a coordinate frame rotating rigidly around the origin with angular velocity $\Omega=-1$, the velocity becomes radial, and thus parallel to the magnetic field (which is not changed by the coordinate transformation). It can be proved that in this rotating frame the magnetic field and the velocity field are aligned everywhere, if they are aligned at the boundary and if the flow is stationary. This is again just a consequence of the MHD frozen-in condition. In the rest frame, however, the flow is not field-aligned, as can be seen in Fig. 7. This flow can thus be interpreted as a field-aligned radial outflow from a rotating object and is related to flows studied in the context of stellar winds [26, 42].

We want to study the grid convergence behavior of this non-field-aligned flow, but cannot directly use the characteristic invariants derived for field-aligned flow. Fortunately, related invariants can be found for this effectively 1D rotating flow [26, 42], as is briefly reviewed next.

The rotating outflow is completely specified when the six constants $s, f_{\mathrm{m}}, f_{\mathrm{B}}, \Omega, r_{\mathrm{A}}$, and $h$ are chosen in

$$
\begin{aligned}
s & =\frac{p}{\rho^{\gamma}}, \\
f_{\mathrm{m}} & =\rho v_{r} r, \\
f_{\mathrm{B}} & =B_{r} r,
\end{aligned}
$$




$$
\begin{gathered}
\left(v_{\theta}-\Omega r\right) B_{r}-v_{r} B_{\theta} \equiv E_{z} \equiv 0, \\
\Omega r_{\mathrm{A}}^{2}=r\left(v_{\theta}-\frac{B_{r} B_{\theta}}{\rho v_{r}}\right) \equiv L, \text { and } \\
h=\frac{1}{2} v_{r}^{2}+\frac{1}{2}\left(v_{\theta}-\Omega r\right)^{2}+\frac{\gamma}{\gamma-1} \frac{p}{\rho}-\frac{1}{2} \Omega^{2} r^{2} .
\end{gathered}
$$

Here $s$ is the entropy, $f_{\mathrm{m}}$ and $f_{\mathrm{B}}$ are the radial momentum flux and magnetic flux, $\Omega$ is the angular speed of the rotating object, $r_{\mathrm{A}}$ is the Alfvén radius, and $h$ is the Bernoulli constant. $E_{z}$ is the electric field in the $z$ direction, and $L$ is the angular momentum density. The invariants defined in Eq. (18) are used to study grid convergence for the rotating outflow problem in Section 6.

Following Sakurai [42], we can derive the Bernoulli function $H(r, \rho)$,

$$
H(r, \rho)=\frac{f_{\mathrm{m}}^{2}}{2 \rho^{2} r^{2}}+\frac{1}{2} r_{\mathrm{A}}^{2} \Omega^{2}\left(\frac{r_{\mathrm{A}} / r-r / r_{\mathrm{A}}}{1-\rho f_{\mathrm{B}}^{2} / f_{\mathrm{m}}^{2}}\right)^{2}+\frac{\gamma}{\gamma-1} s \rho^{\gamma-1}-\frac{1}{2} \Omega^{2} r^{2}
$$

For given constants $s, f_{\mathrm{m}}, f_{\mathrm{B}}, \Omega$, and $r_{\mathrm{A}}$ we can implicitly describe the orbits $\rho(r)$ as level curves of the Bernoulli function $H(r, \rho)=h$ for varying $h$. Figure 8 shows these orbits (dotted) for the values of $s, f_{\mathrm{m}}, f_{\mathrm{B}}, \Omega$, and $r_{\mathrm{A}}$ corresponding to the simulation shown in Fig. 7. The thick solid orbit corresponds to the value of the Bernoulli constant $h$ of the simulation. Two other curves of interest are the fast/slow Mach curve defined by $\partial H / \partial \rho=0$ (thin solid) and the throat curve defined by $\partial H / \partial r=0$ (dashed). Orbits are vertical where they cross the fast/slow Mach curve, and horizontal where they cross the throat curve. The fast/slow Mach curve and the throat curve cross at an O-type critical point. The other critical point is located at infinity. This critical point analysis is instructive because it shows that the

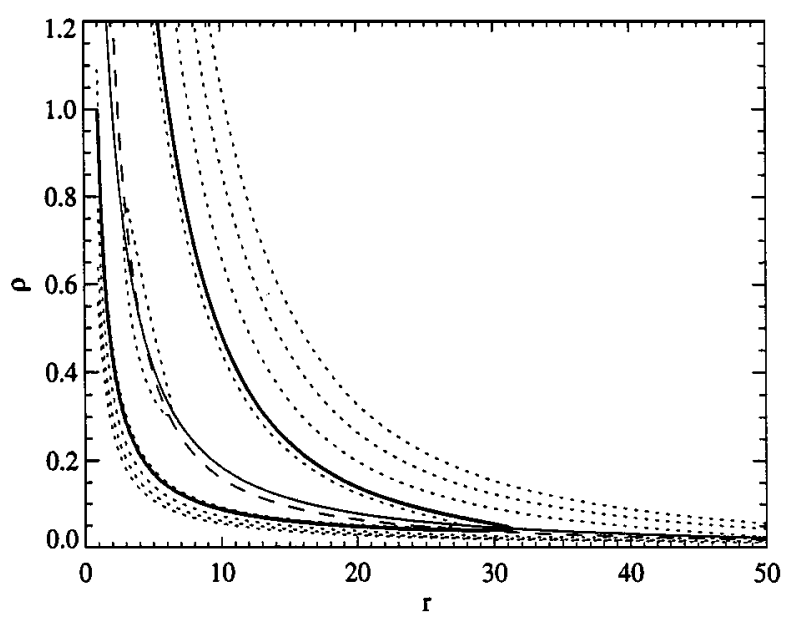

FIG. 8. Critical point analysis for the rotating outflow problem. The dotted lines are solution orbits. The thick solid line is the orbit corresponding to the flow shown in Fig. 7. The thin solid line is the fast/slow critical curve, and the dashed line is the "throat" curve. There is one finite critical point (of O-type) where these two curves intersect. The outer radius of the simulation domain has to be chosen smaller than approximately $r=30$ to obtain a continuous stationary solution. Indeed, at the point where the orbit and the fast/slow line cross, the flow becomes subfast. The continuous orbit becomes multi-valued, which means that there is no continuous solution, but a (non-stationary) shock would be formed. 
outer radius of the simulation domain has to be chosen smaller than approximately $r=30$ to obtain a continuous stationary solution. Indeed, at the point where the orbit and the fast/slow line cross, the flow becomes subfast. The continuous orbit becomes multi-valued, which means that there is no continuous solution, but a (non-stationary) shock would be formed. The model problem of Fig. 7 does thus not constitute an example of a smooth outflow in an infinite domain. In an appropriately chosen finite domain, however, this problem describes a valid MHD flow with well-defined boundary conditions (the flow remains superfast at the outer boundary), and is thus perfectly suitable for the purpose of testing a numerical MHD code through grid convergence study. To conclude, we can remark that this non-field-aligned model problem is really a very special case. It could be constructed from the cylindrical expansion flow by rotation because the cylindrical expansion problem has the peculiar property that the flow, the boundary shapes, and the boundary conditions themselves are all rotationally invariant. We anticipate that it will be very difficult to find stationary 2D non-field-aligned model problems without such special symmetries.

\section{A FINITE VOLUME NUMERICAL SCHEME}

In this section we discuss the standard 2D high-resolution finite-volume numerical MHD scheme on a structured body-fitted grid for which we investigate grid convergence of the presented model flows further on in the paper. Our discussion is brief because most of the numerical techniques have been presented before [e.g., 1, 28, 32, 33, 36, 57] and because the presentation of this numerical scheme is not the main motivation of this paper. We intend to give a description which completely specifies our numerical approach, however, because this establishes the repeatability of our numerical experiments and will facilitate comparison with other numerical codes [11]. This code has been implemented for use on massively parallel computers using the MPI message passing library. We describe the 2D version of the code here, but a $3 \mathrm{D}$ version has been implemented as will using basically the same algorithms [33, 47].

The ideal MHD equations (1) can be written in the following abstract conservation law form,

$$
\frac{\partial \mathbf{U}}{\partial t}+\nabla \cdot \mathbf{F}(\mathbf{U})=\mathbf{S},
$$

with $\mathbf{U}$ the vector of state variables which are conserved quantities, $\mathbf{F}$ the flux vector, and $\mathbf{S}$ the Powell source term.

\subsection{Spatial Discretization}

We divide the computational domain into a logically rectangular structured grid of quadrilaterals. The solution of the flow is sought in the physical cells, with indices in the computational domain ranging from 1 to $n_{i}$ for index $i$, and from 1 to $n_{j}$ for index $j$. This physical domain is surrounded by two layers of ghost cells, which allow for a simple implementation of boundary conditions (see Section 5.5). The cell interfaces are not constrained to be parallel to a Cartesian axis, which for instance allows to fit the grid to a curved rigid body. We integrate Eq. (20) formally over the cell with label $(i, j)$, and obtain the discretized 
equation

$$
\frac{\partial \overline{\mathbf{U}}_{i, j}}{\partial t}+1 / \Omega_{i, j} \sum_{k=1}^{4} \mathbf{F}_{k}^{*} \cdot \boldsymbol{n}_{k} l_{k}=0,
$$

for the time evolution of the average of the state variable over cell $(i, j)$

$$
\overline{\mathbf{U}}_{i, j}=\left(\iint \mathbf{U}(x, y, t) d x d y\right) / \Omega_{i, j},
$$

which is stored in the center of cell $(i, j)$. Here $\Omega_{i, j}$ is the area of cell $(i, j)$, and the summation in Eq. (21) extends over the four sides or interfaces of cell $(i, j) . \mathbf{F}_{k}^{*}$ is a numerical approximation for the flux vector through the interface $k, l_{k}$, is the length of interface $k$, and $\boldsymbol{n}_{k}$ is the outward unit vector normal to side $k$.

For each interface, we use a numerical flux function of the form

$$
F^{*}\left(\mathbf{U}_{l}, \mathbf{U}_{r}\right)=\frac{F\left(\mathbf{U}_{l}\right)+F\left(\mathbf{U}_{r}\right)}{2}+D\left(\mathbf{U}_{l}, \mathbf{U}_{r}\right),
$$

to calculate $F^{*}=\mathbf{F}^{*} \cdot \boldsymbol{n}$. Here $F$ is the 1D MHD flux function of Eq. (7), and $\mathbf{U}_{l}$ and $\mathbf{U}_{r}$ are the state variables to the left and to the right of the interface. The third term, $D\left(\mathbf{U}_{l}, \mathbf{U}_{r}\right)$, is in general proportional to $\mathbf{U}_{r}-\mathbf{U}_{l}$. A first-order accurate spatial discretization is obtained if we take the left and the right state used to calculate the numerical flux to be the cellaverages to the left and the right of the interface. In this picture, the solution is imagined piecewise constant in every cell. The third term $D\left(\mathbf{U}_{l}, \mathbf{U}_{r}\right)$ adds an amount of (numerical) dissipation appropriate to make the scheme numerically stable. There are many choices for the exact form of this dissipative term, corresponding to the choice of an (approximate) Riemann solver. This is discussed in Section 5.4. Second-order spatial accuracy is obtained by considering piecewise-linear variation in a cell. The values at the cell interface are then calculated via linear reconstruction. To discard spurious oscillations at discontinuities, we use the non-linear minmod slope limiter [28] to determine the slope of the linear reconstruction, and for robustness reasons we do the second-order reconstruction using the primitive variables W. Experience shows that the accuracy at boundaries is improved when the nonlinear limiting is performed on vector components in the coordinate system aligned with the interface. This simple dimension-by-dimension approach turns out to work well, and numerical experiments like the ones to be shown further on in this paper show that the scheme remains close to second-order accurate if the grid is not distorted too much. A more sophisticated approach would be to do reconstruction using estimates of gradients based on $2 \mathrm{D}$ interpolation [5].

\subsection{Discretization of the Source Term}

We use the following discretization for the source term $\mathbf{S}_{i, j}$ in cell $(i, j) . \nabla \cdot \boldsymbol{B}$ is discretized as

$$
(\nabla \cdot \boldsymbol{B})_{i, j}=1 / \Omega_{i, j} \sum_{k=1}^{4} \boldsymbol{B}_{k} \cdot \boldsymbol{n}_{k} l_{k}
$$


with

$$
\boldsymbol{B}_{k}=\left(\boldsymbol{B}_{l}+\boldsymbol{B}_{r}\right) / 2,
$$

the average of the (reconstructed) magnetic fields on the left and the right of the interface. This discretization of $\nabla \cdot \boldsymbol{B}$ is then multiplied with the appropriate cell-averaged state variables (stored in the center of cell $(i, j)$ ) to obtain a discretization for the source term of Eq. (1). Experience has shown that it is important to use the reconstructed values in the discretization of $\nabla \cdot \boldsymbol{B}$ for the second-order scheme, because this seems to result in a more robust scheme.

\subsection{Temporal Discretization}

We use explicit Runge-Kutta time integrators. A one-stage scheme is used for the firstorder scheme, and a two-stage scheme for the second-order scheme [32,33]. The time-step $\Delta t$ is derived from the CFL-like time-step limitation [33]

$$
\Delta t=c_{\mathrm{CFL}} \min _{i, j}\left[\frac{\Omega_{i, j}}{\sum_{k=1}^{4} \max \left(0,\left(\boldsymbol{v}_{i, j} \cdot \boldsymbol{n}_{k}+c_{k, i, j}^{f}\right)\right) l_{k}}\right],
$$

with $c_{k, i, j}^{f}$ the fast MHD wave speed in the direction $\boldsymbol{n}_{k}$ calculated with the cell-averaged state values stored in cell $(i, j)$. The constant $c_{\mathrm{CFL}}$ has to be chosen smaller than one for the first-order scheme, and may be chosen slightly larger than one for the second-order scheme. We use $c_{\mathrm{CFL}}=0.8$ for all the calculations presented further on in this paper.

We use these general time-accurate integration methods to calculate the stationary flow solutions to be described further on in this paper. In general, we start from a uniform initial flow condition and we evolve the flow in time until vanishing of the residuals $\mathbf{R}$ shows that a steady state has been reached. We use the following quantity based on the density residual to measure the convergence to a steady state at iteration $m$,

$$
\bar{R}(m)=\log \left(c_{\text {norm }} \sqrt{\left(\frac{\sum_{i, j}\left(R_{i, j}^{\rho}(m)\right)^{2}}{n_{i} n_{j}}\right)}\right),
$$

with $n_{i}$ and $n_{j}$ the number of cells in the $i$ and $j$ direction, $R_{i, j}^{\rho}(m)$, the density residual in cell $(i, j)$, and the normalization constant $c_{\text {norm }}$ chosen in such a way that $\bar{R}(0)=0$. We routinely achieve convergence of 15 orders of magnitude, which means that the residuals are driven to machine zero. Throughout this article we mean the base-10 logarithm when we use "log" in convergence measures and on plots.

It would be possible to obtain convergence to a steady state more efficiently, and many convergence acceleration methods of varying complexity could be tried, ranging from simple local time-stepping over implicit residual smoothing and multigrid to fully implicit time integration $[33,54]$, but simple explicit time integration is sufficient for our study of grid convergence of the spatial discretization.

\subsection{Numerical Flux Functions}

Throughout the years, many interesting MHD numerical flux functions have been proposed that can be used in the type of finite volume discretization described above, most of 
them based on some kind of approximate Riemann solver at the interface between two cells $[1,4,5,8,12-14,17,34,36,39-41,55,58]$. Many of those flux functions are designed to produce as sharp as possible shock transitions and tangential discontinuities. The Roe scheme, for example, which has been very popular for hydrodynamic applications, has been extended to MHD [5, 8, 36, 39]. This scheme tries to minimize the numerical dissipation by decomposing the difference $\mathbf{U}_{r}-\mathbf{U}_{l}$ present in $D\left(\mathbf{U}_{l}, \mathbf{U}_{r}\right)$ in the space of the eigenvectors of the Jacobian and by applying the minimum amount of numerical dissipation to every characteristic wave separately. There remain, however, several serious problems of local numerical instability with this scheme, like the carbuncle phenomenon, as for example described by Quirk [37]. Probably because of these problems the Roe scheme is not much used in MHD simulations [26, 32, 45].

Several approaches have been proposed to remedy these problems. First, different types of non-linear flux functions are being investigated, e.g., Linde's HLLE-based solver for MHD [31], which is based on some other form for the numerical dissipation, or solvers derived from kinetic descriptions [31,32]. These new flux functions seem to remedy some of the problems associated with the Roe solver, but more investigation is necessary to see if they can solve all the stability problems. Second, it is sometimes argued that much of the problems with finite volume schemes on structured grids are inherent to the dimension-bydimension approach, and that many of the pathological instabilities could be removed by considering truly multi-dimensional schemes on unstructured grids [11]. Third, the failure of the Roe scheme can probably be related to the fact that it is not entropy-stable [5]. New entropy-stable schemes formulated in symmetrized entropy variables are being developed [5] and it can be expected that they will lead to more stable numerical schemes. Also in this area much research is still going on.

This short discussion shows that there are certainly many unsolved issues regarding the choice of numerical flux functions and numerical schemes in general. In this paper, we make the "conservative" choice to carry out this grid convergence study using the (local) Lax-Friedrichs flux function $[4,28,55]$, which is given by

$$
F^{*}\left(\mathbf{U}_{l}, \mathbf{U}_{r}\right)=\frac{F\left(\mathbf{U}_{l}\right)+F\left(\mathbf{U}_{r}\right)}{2}-\left(\left|v_{\mathrm{n}}^{*}\right|+c_{\mathrm{n}}^{f *}\right) \frac{\mathbf{U}_{r}-\mathbf{U}_{l}}{2},
$$

with $\left|v_{\mathrm{n}}^{*}\right|+c_{\mathrm{n}}^{f *}$ the largest wave speed in the direction normal to the interface, determined from the arithmetic average $\left(\mathbf{U}_{l}+\mathbf{U}_{r}\right) / 2$ on the interface. The Lax-Friedrichs flux function applies to all characteristic waves the same numerical dissipation, determined by the maximum wave speed, which makes it more dissipative than the Roe scheme, for instance, but much more robust and less prone to local numerical instabilities. The Lax-Friedrichs flux function is certainly one of the most robust and simple numerical flux functions. For these reasons its use in MHD applications is often advocated [4, 26, 45, 55]. Stationary shock profiles are actually captured surprisingly well with the Lax-Friedrichs scheme [4, 55], but tangential discontinuities are smeared out. We show further on that we obtain satisfactory grid convergence results using this numerical flux function. It will be interesting to test if other flux functions lead to acceptable grid convergence results as well, but such an extensive comparison is outside the scope of this paper. One of the main purposes of this paper is to show how grid convergence of MHD codes can be investigated in principle, and we have made the deliberate choice to illustrate this using the most robust, simple, and reliable MHD numerical flux function, and not one of the other flux functions which are more prone to local numerical instabilities. 


\subsection{Boundary Conditions}

We implement boundary conditions making use of two layers of ghost cells (e.g., [29]). For the model flows presented, we need three types of boundary conditions. First, at perfectly conducting walls, the magnetic and velocity fields have to be tangent to the wall. This is implemented by copying the cell-averaged state of the last physical cell into the first ghost cell with the magnetic and velocity fields mirrored relative to the boundary segment. The next-to-last physical cell is copied into the second ghost cell with mirrored magnetic and velocity fields. Second, at free outflows, where the normal outward plasma velocity is larger than the normal fast MHD wave speed and all the characteristic information thus propagates outward of the physical domain, the state variables of the last two physical cells are used to extrapolate linearly into the two layers of ghost cells. Third, at free inflows, where the normal inward plasma velocity is larger than the normal fast MHD wave speed and all the characteristic information thus propagates into the physical domain, we impose the value on the boundary interface and use this and the state of the last physical cell to calculate the value in the ghost cells using linear interpolation. Although these boundary conditions are again based on a simple dimension-by-dimension extrapolation, they turn out to work well for the model flows to be discussed below, which have only moderately deformed grids. We have also tested different types of characteristic boundary conditions for the inflows and outflows not based on ghost cells [5], and flux boundary conditions for the perfect walls, but for these different implementations of boundary conditions we found essentially the same grid convergence behavior. Therefore we have chosen to present the results with the more simple and standard ghost cell approach.

\subsection{Grid Convergence}

We are now at a point where we can define grid convergence criteria for stationary model flows, but we first discuss what kind of convergence order we can expect for model flows simulated with the numerical techniques discussed above.

5.6.1. Formal grid convergence. The basic idea of grid convergence is that for smooth flow (see below), some measure of the error of the simulation result should decrease as a function of the resolution with an order which is the formal order of accuracy of the scheme. Formal Taylor series expansion of the numerical schemes presented above would show that for a stationary solution

$$
E=\|\mathbf{U}-\overline{\mathbf{U}}\|=c(1 / n)^{p},
$$

with $\mathbf{U}$ the exact solution, $\overline{\mathbf{U}}$ the numerical solution, $\boldsymbol{E}$ the error in some norm, $c$ a constant, $n$ the number of cells in a certain direction-where it is understood that the resolution is changed proportionally in all directions - and $p$ the order of the method ( 1 or 2 for the schemes discussed above). On logarithmic axes this would lead to grid convergence curves which are straight lines with slope 1 or 2 :

$$
\log (E)=\log (c)-p \log (n)
$$

All state variable quantities are expected to be calculated with the accuracy of the scheme. It is important to remark here, however, that the $\nabla \cdot \boldsymbol{B}$ quantity ("magnetic flux production per unit volume") with discretization given by Eq. (24) is a sum of derivatives of the 
magnetic field state variables, and can thus be expected to converge more slowly than the state variables, in the worst case with one order less [5, 16]. Probably for this reason, Linde $[31,36]$ proposes to measure magnetic flux conservation in a different way. The alternative quantity

$$
F_{i, j}=\frac{\sum_{k=1}^{4} \boldsymbol{B}_{k} \cdot \boldsymbol{n}_{k} l_{k}}{\sum_{k=1}^{4} l_{k}}
$$

is a measure of magnetic flux conservation ("flux production per unit length") which should converge at least with the same order as the state variables. We investigate how these two measures of flux conservation behave for the numerical simulation of our five model flows.

The actually observed convergence order for a numerical simulation can be lower than the formal order of accuracy of the scheme for several reasons. We discuss in short five possible reasons for convergence degradation. The first two reasons are related to the analytical properties of fluid flows, viz., to the regularity of flows. The latter three reasons for convergence degradation seem to be more related to the details of the numerical scheme.

First, when the analytical solution to a flow contains (weak) discontinuities, then the derivatives in the above-mentioned Taylor expansion do not exist everywhere, which means that the convergence order result derived using the Taylor expansion is not valid. In general it can be expected that the convergence order for a numerical scheme will be lower for a solution containing discontinuities. Leveque [28] gives an example where the convergence order of an approximation degrades by 0.5 for a solution containing a discontinuity.

Second, near geometrically singular points on boundaries, the analytical solution to the flow problem is generally not smooth, with similar consequences of convergence-order degradation [43]. These effects can be reduced by choosing smooth boundaries, as we have done for all our model flows, but even then the finite grid resolution leads to singular corners at curved boundaries. The effects of these singularities can be reduced by a careful geometrical refinement of the grid near the boundary [43]. Barth [5] reports improved grid convergence if interpolation is done with higher order polynomials at the boundary interfaces than inside the domain (so-called iso-parametric boundaries), in the context of finite element methods which allow more flexibility than our finite volume schemes.

Third, the choice of numerical flux function seems to be important for the errors induced at boundaries. For instance, it is well known that the Roe scheme can lead to the problem of wall-heating at perfect wall boundaries [29]. It seems that our choice of the Lax-Friedrichs flux function in general performs better in such situations, and our grid convergence results seem generally to be satisfactory. We have thus not adopted the strategy to exclude physical cells close to the boundaries from our error norm calculations $[49,50]$, because we think that this process is somewhat artificial and arbitrary, and because we want to prove grid convergence of our standard numerical scheme with the boundary treatment included. We have to admit, however, that the errors are often large not only at discontinuities, but also at the boundaries.

Fourth, near discontinuities our second-order scheme will switch to first-order accuracy due to the action of the non-linear limiter. In error norms calculated over the whole simulation domain, these local first-order errors dominate the second-order errors in smooth parts of the flow, resulting in convergence degradation to first order.

Fifth, simulations on highly distorted grids, with angles in cells substantially deviating from $90^{\circ}$ or cells highly elongated, may show degraded convergence rates [56]. 
5.6.2. Practical grid convergence criteria. For the model flows we propose in this article, the analytical solution is generally not known. How can we carry out grid convergence studies then? We do not engage in the use of "self-convergence" criteria $[49,50]$, because they do not easily lead to rigorous conclusions.

But even when the analytical solution is not known, we can formulate grid convergence criteria. In general we can distinguish three classes of grid convergence criteria.

The first class of criteria follows directly from the divergence nature of the steady conservation law (Eq. (1) with vanishing time derivative) and the $\nabla \cdot \boldsymbol{B}$ constraint. These laws basically state that the divergence of a flux vanishes. This leads to grid convergence criteria in two ways.

First, over the whole physical domain, the integrated form of the divergence law shows that the line integral of the normal flux through the circumference of the physical domain has to vanish. For instance, in a steady flow the net flux of density (the momentum) through the boundaries has to vanish, and numerically this net flux has to converge to zero in function of resolution. This, however, merely checks global conservation and only gives a very global measure of accuracy, as internal errors can cancel out, and this grid convergence measure is not further exploited in this article.

Second, when the flux vectors are aligned with a boundary at two opposing boundaries of the simulation domain, and the boundaries thus form flux tubes, the flux through any line connecting the two boundaries has to be the same. This can be tested along lines consisting of cell interfaces. We extensively use this observation to verify if the magnetic and momentum fluxes through sections of flux tubes are constant along the flux tubes. This leads to a more local measure of accuracy. Suppose for instance that the boundaries below $j=1$ and above $j=n_{j}$ are perfect walls; then we can calculate the (magnetic or momentum) flux $\Phi_{i+1 / 2}$ through every line formed by interfaces between cells with equal coordinates $i$ and $i+1$, using the reconstructed $\mathbf{U}_{l}$ and $\mathbf{U}_{r}$ in the average $\left(\mathbf{U}_{l}+\mathbf{U}_{r}\right) / 2$ at every interface, and with $i$ running from 0 to $n_{i}$. Then an L1 error measure can be defined as

$$
E_{\Phi}^{\mathrm{B}, \mathrm{m}}=\frac{\sum_{i=0}^{i=n_{i}} \operatorname{abs}\left(\Phi_{i+1 / 2}-\Phi_{\text {theor }}\right)}{n_{i}+1},
$$

with $\Phi_{\text {theor }}$ the known theoretical value of the flux, and $E_{\Phi}^{\mathrm{B}}$ and $E_{\Phi}^{\mathrm{m}}$ the magnetic and momentum flux error measure, respectively. Flux conservation criteria such as this have been used before to investigate the accuracy of MHD simulations in function of resolution $[1,31,49,50]$.

The second class of grid convergence criteria follows from the rigorously defined characteristic properties of the class of flows under consideration and leads to measures of true local grid convergence in every cell of the simulated flow. There can be up to four global invariants for stationary planar field-aligned flow, as follows from the characteristic analysis. The angle $\theta$ between the magnetic and the velocity fields has to vanish everywhere. In the case of uniform inflow, the Riemann invariants $\rho / \alpha$ and $h_{s}$ are global invariants. If the flow is additionally smooth, then the Riemann invariant $s$ is a fourth global invariant. An L1 error measure for these invariants $I$ can be defined as

$$
E_{I}=\frac{\sum_{i, j} \operatorname{abs}\left(\bar{I}_{i, j}-I_{\text {theor }}\right) \Omega_{i, j}}{\sum_{i, j} \Omega_{i, j}},
$$


with $I_{\text {theor }}$ the known theoretical value of the invariant. Note that we actually calculate the difference between the cell-averaged numerically calculated values of the invariant and the cell average of the theoretical value of the invariant (which, of course, is the invariant itself). Global grid convergence criteria based on characteristic invariants have to our knowledge not often been used to investigate the accuracy of 2D MHD simulation results in function of resolution. The $s$ and $h_{s}$ quantities keep their invariant properties in stationary Euler flow, so this type of grid convergence study can also be carried out for that case, as is well known. Finally, we should mention that $\nabla \cdot \boldsymbol{B}$ should vanish in theory for every MHD flow, so this can easily be tested using the same expression to calculate the error.

The third class of grid convergence criteria follows from the RH jump relations. If sufficient information is available in terms of imposed upstream flow conditions and geometrical constraints, then the remaining unknown values can be calculated from Eq. (6) with high accuracy, and in some cases analytical solutions can be found. These values can then be compared with the values resulting from full 2D numerical simulations of the flow, and grid convergence can be investigated.

In practice, we measure the simulation error using the above given expressions $E$ as a function of the number of grid cells in a certain direction $n$ and determine the numerical convergence order by a least-squares fit of the $\log$ E- $\log n$ curve with generally four data points.

\section{GRID CONVERGENCE STUDY OF MHD MODEL FLOWS}

In this section we discuss numerical simulation aspects of the model flows presented in Section 3 and the non-field-aligned flow presented in Section 4, and we present grid convergence results obtained with the criteria discussed in Section 5.

\subsection{Numerical Simulation Aspects}

The steady state simulation results shown in Figs. 2-6 are obtained via time-accurate relaxation starting from uniform initial states. Figure 9 shows the convergence of the logarithm of the density residual towards a steady state solution as a function of the number of time-steps. We can observe that the steady state convergence of our numerical scheme is extremely well behaved. For all simulations we obtain convergence up to machine accuracy using both the first-order scheme (solid) and the second-order scheme (dash-dotted). The number of time-steps needed to obtain convergence is mostly similar for the first- and the second-order schemes. We used the same CFL number for the first- and the second-order scheme. The computational cost per time-step is, however, about three times higher for the second-order scheme. The number of time-steps is much higher for the bow shock flow than for the other flows, because of the low speeds in the elliptic region near the stagnation point.

Figure 10 shows the simulation grids used for the numerical results presented in this paper. The grids are nearly uniform and the grid cells are mostly quite regular, except perhaps for some strongly deformed cells in the bow shock grid. For the cylindrical expansion problem and the rotating outflow problem the grid convergence has been studied making use of simulations on $40 \times 40,50 \times 50,60 \times 60$, and $70 \times 70$ grids. The expanding tube problem has been simulated on $30 \times 50,45 \times 75,60 \times 100$, and $75 \times 125$ grids, and the wedge problem on $40 \times 80,60 \times 120,80 \times 160$, and $100 \times 200$ grids. Grid convergence for the bow shock flow has been studied making use of simulations on $20 \times 20,40 \times 40,60 \times 60$, 
(a) cylindrical expansion

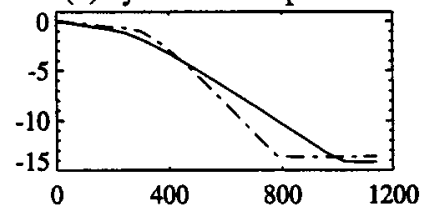

(c) wedge

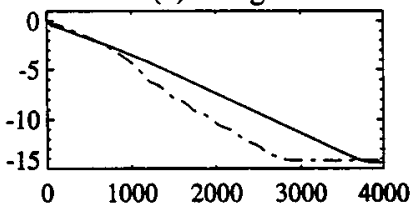

(e) nozzle

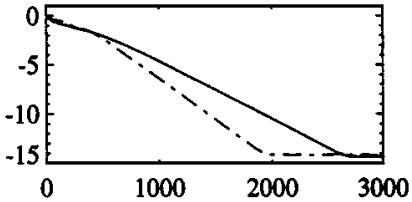

(b) expanding tube

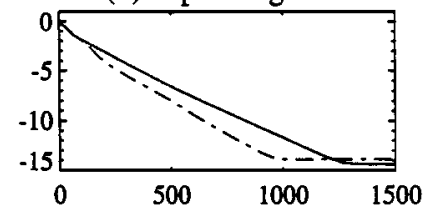

(d) bow shock

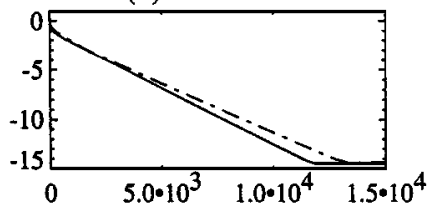

(f) rotating outflow

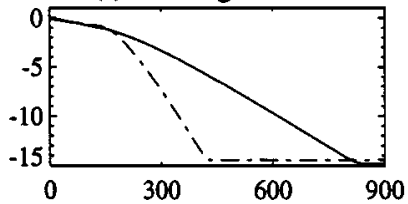

FIG. 9. Convergence of the logarithm of the density residual towards a steady state, in function of the number of time-steps. First-order (solid) and second-order (dash-dotted) numerical schemes.

and $80 \times 80$ grids, and the nozzle simulations were performed on $48 \times 16,72 \times 24,96 \times$ 32 , and $120 \times 40$ grids.

\subsection{Grid Convergence Results for Flux Conservation}

The cylindrical expansion flow, the expanding tube flow, the wedge flow, and the nozzle flow all have opposed simulation domain boundaries which are ideal walls and thus define
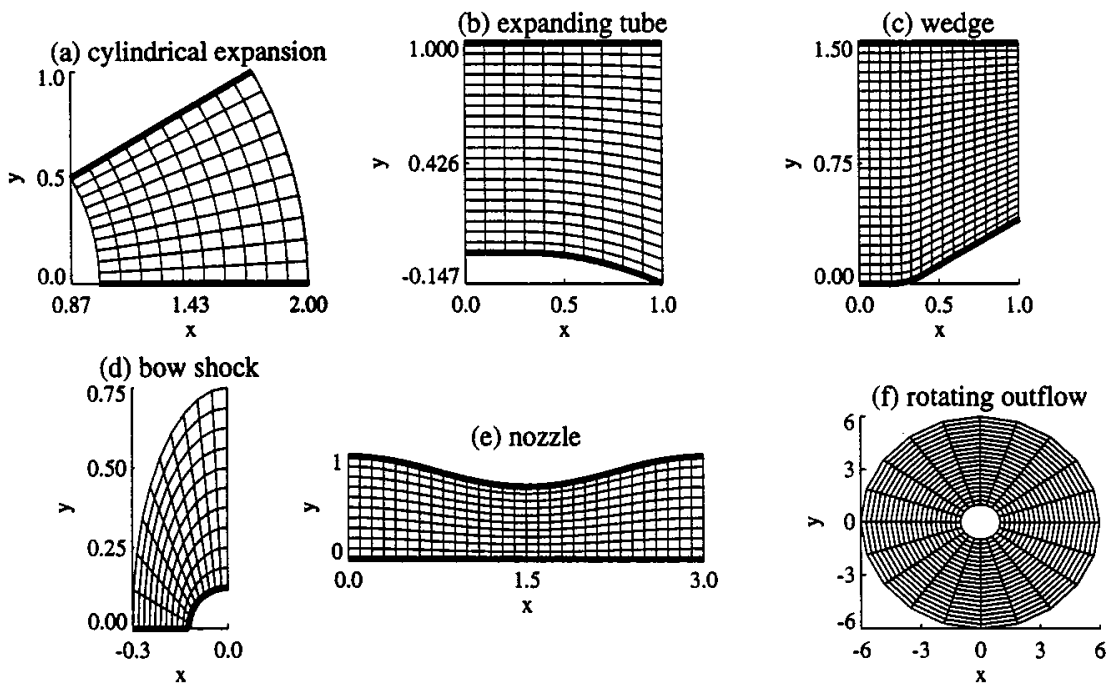

FIG. 10. Finite-volume simulation grids. Successive conformal refinements of these grids have been used for the grid convergence study. 
(a) cylindrical expansion (b) expanding tube
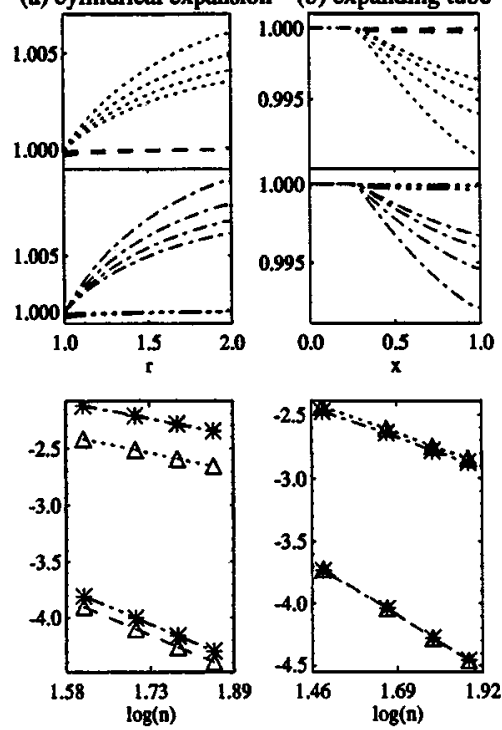
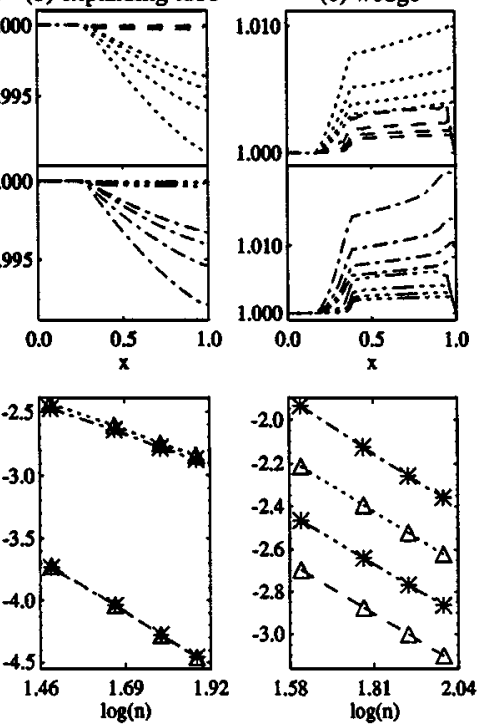

(c) wedge

(d) nozzle
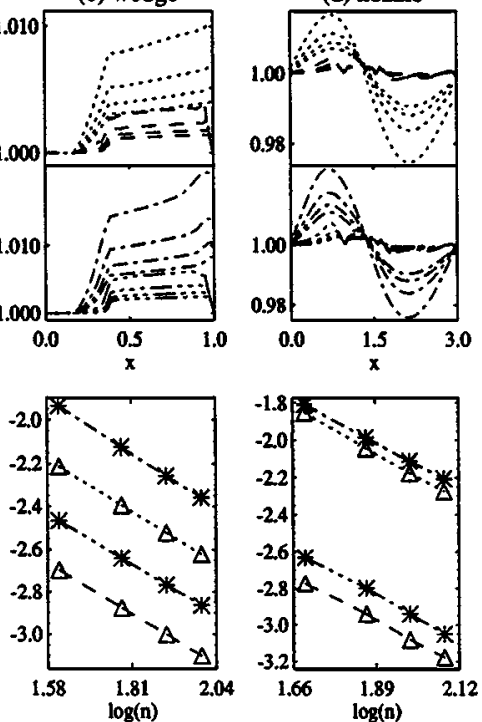

(e) rotating outflow
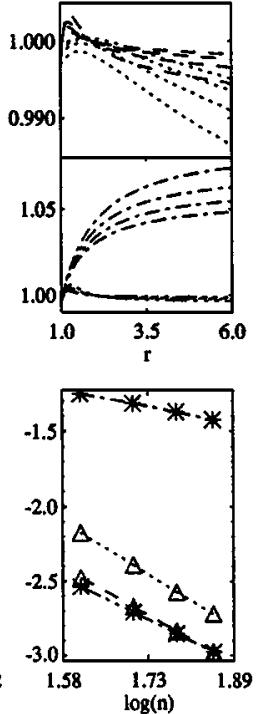

FIG. 11. Grid convergence behavior of the magnetic and momentum flux conservation for five test cases. The first row shows the normalized magnetic flux through vertical or radial sections in function of the vertical or radial coordinate for four different grid resolutions, both for the first-order scheme (dotted) and the secondorder scheme (dashed). The second row shows the normalized momentum flux profiles for the first-order scheme (dash-dotted) and the second-order scheme (dash-dot-dot-dotted). The third row shows the 10 logarithm of the L1 norm of the errors of the magnetic flux (triangles, first-order dotted and second-order dashed) and momentum flux (asterisks, first-order dash-dotted and second-order dash-dot-dot-dotted), in function of the grid resolution (base 10 logarithms of $n$, the number of grid cells in the $i$ direction).

a flux tube. We can study grid convergence of the magnetic and momentum flux through these flux tubes. The rotating outflow problem described in Section 4 has the property of radial conservation of magnetic and momentum flux. Figure 11 shows the grid convergence behavior of the fluxes for these five test cases.

The first row of the figure shows the normalized magnetic flux through vertical or radial sections in function of the vertical or radial coordinate. Every panel contains eight curves, four for the first-order scheme (dotted) for increasing grid resolution and four for the secondorder scheme (dashed). We see that in all cases the normalized magnetic flux approaches the value of unity nicely and that the flux conservation is much more accurate for the second-order results than for the first-order results.

The second row of the figure shows the normalized momentum flux through the vertical or radial sections. Every panel again contains eight curves, four for the first-order scheme (dash-dotted) for increasing grid resolution, and four for the second-order scheme (dashdot-dot-dotted). We again see that in all cases the normalized momentum flux approaches the value of unity nicely and that the flux conservation is much more accurate for the second-order results than for the first-order results.

The shape of the momentum flux curves is generally similar to the shape of the magnetic flux curves, and this is no surprise for field-aligned flow. Only for the non-field-aligned rotating outflow (Fig. 11e) are the curves markedly different in shape.

The third row shows the base-10 logarithms of the L1 norms of the errors of the magnetic and momentum flux curves, as functions of the grid resolution (base-10 logarithms of $n$, the number of grid cells in the $i$ direction). The magnetic flux conservation for the first-order 


\section{TABLE I}

Grid Convergence Order of Several Invariant Quantities

\begin{tabular}{lcccccc}
\hline & Expansion & Tube & Wedge & Bow shock & Nozzle & Rotating \\
\hline \multicolumn{7}{c}{ First order } \\
Magnetic flux & -0.98 & -1.05 & -1.04 & & -1.08 & -2.30 \\
Momentum flux & -0.93 & -1.04 & -1.08 & & -1.01 & -0.72 \\
$s$ & -0.93 & -0.87 & & & -0.81 \\
$h_{s}$ & -0.82 & -1.04 & -1.15 & -0.86 & -0.91 & $-0.58^{*}$ \\
$\rho / \alpha$ & -0.87 & -0.99 & -0.97 & -0.72 & -0.70 & $-0.82^{*}$ \\
$\theta$ & & -0.99 & -1.06 & -0.60 & -0.73 & $-0.84^{*}$ \\
$\nabla \cdot \boldsymbol{B}$ & -0.98 & -0.92 & -0.79 & -0.59 & -0.90 & -1.33 \\
$F=\sum\left(B_{n i} \Delta l_{i}\right) / \sum \Delta l_{i}$ & -1.98 & -1.92 & -1.79 & -1.55 & -1.90 & -2.36 \\
& & Second & & & \\
Magnetic flux & -2.06 & -1.90 & -1.03 & & -1.04 & -2.10 \\
Momentum flux & -2.06 & -1.86 & -1.02 & & -1.07 & -1.85 \\
$s$ & -1.96 & -1.74 & & & -1.28 \\
$h_{s}$ & -1.91 & -1.10 & -1.01 & -0.90 & -0.87 & $-1.92^{*}$ \\
$\rho / \alpha$ & -2.07 & -1.44 & -0.82 & -1.07 & -0.97 & $-1.71^{*}$ \\
$\theta$ & & -1.78 & -0.99 & -0.85 & -1.01 & $-1.89^{*}$ \\
$\nabla \cdot \boldsymbol{B}$ & -1.96 & -1.38 & -0.22 & -0.55 & -0.42 & -1.79 \\
$F=\sum\left(B_{n i} \Delta l_{i}\right) / \sum \Delta l_{i}$ & -2.95 & -2.39 & -1.22 & -1.53 & -1.43 & -2.83 \\
\hline & & & & & & \\
\hline
\end{tabular}

Note. The fitted slope of the base 10 logarithms of the L1 norm of the error in function of the 10 logarithms of the number of grid cells in the $i$ direction is shown. For the values marked with an asterisk, the rotating outflow grid convergence is measured using slightly different invariant quantities than for the other flows. The results show that the presence of discontinuities (in the wedge, bow shock, and nozzle flows) consistently degrades the convergence for the second-order numerical scheme to first order due to the action of the nonlinear limiter. Analytical singularities or numerical inaccuracies at boundaries and grid distortion may further degrade the convergence. Such additional convergence degradation can be observed for the values that are italicized. Overall, a satisfactorily consistent grid convergence behavior is obtained.

scheme is indicated by a dotted line with triangles and by a dashed line with triangles for the second-order scheme. The momentum flux conservation for the first-order scheme is indicated by a dash-dotted line with asterisks and by a dash-dot-dot-dotted line with asterisks for the second-order scheme. We observe that in all cases the convergence curves follow a straight line, which indicates convergence with a well-defined order. The fitted slope coefficients for these lines are presented in Table 1. The cylindrical expansion flow and the expanding tube flow are both smooth flows. The fluxes both converge with the expected slope close to -1 for the first-order scheme and close to -2 for the second-order scheme. The wedge flow contains a strong discontinuity, and due to the action of the nonlinear limiter, the second-order scheme converges only with a slope close to -1 , like the first-order scheme. The second-order results are more accurate, however. The nozzle flow contains shocks as well and also shows first-order convergence both for the first- and the second-order scheme. The rotating outflow problem is smooth and shows the expected second-order convergence for the second-order scheme. For the first-order scheme, the momentum flux converges with an order which is lower than first order, but, remarkably, the magnetic flux converges with slope -2.3 .

The main conclusion to be drawn from this grid convergence study for magnetic and momentum flux conservation is that the experimentally obtained grid convergence orders 
are very close to the theoretically expected orders. The numerical schemes seem thus to behave very well. The excellent conservation of magnetic flux indicates strongly that the Powell source term approach produces valid results.

\subsection{Grid Convergence Results for Global Invariants}

Table 1 contains the fitted slopes of the grid convergence curves for the global invariants entropy $s$, stagnation enthalpy $h_{s}, \rho / \alpha$, and $\theta$. The entropy $s$ is not a global invariant when shocks are present. In the case of the rotating outflow, the invariants are entropy $s$, Bernoulli function $h$, angular momentum $L$, and electric field $E_{z}$, as discussed in Section 4 . Note that no error slope has been given for the grid convergence of the angle $\theta$ between the magnetic and velocity field for the case of the cylindrical expansion. In the initial condition the fields are aligned perfectly up to the machine error induced by a rotation, and this property is conserved throughout the time relaxation as the flow is perfectly radial. These rotational machine errors are very small — much smaller than the errors in the other global RIs — and of course do not depend on resolution, so that for this case grid convergence of $\theta$ is not relevant. The first-order scheme produces grid convergence orders which are generally close to the theoretically expected slope of -1 . For some model problems and for some invariants, the convergence order is smaller than 1 . In this case the slopes are shown in italics, where we have (arbitrarily) put the limit of expected behavior at $80 \%$. Convergence degradation seems to be most severe for the bow shock and nozzle flows, and detailed study of the simulation results shows that this can mainly be attributed to the interaction of the shocks with the boundaries. Overall, however, the results are quite consistent with the order of the scheme. For the smooth flow problems, the second-order scheme produces grid convergence orders which are generally close to the theoretically expected slope of -2 , except for the values in italics. For the expanding tube flow, careful study shows again that convergence degradation can be attributed to interaction with the (not perfectly smooth) boundary. The model problems with shocks show consistent first-order convergence behavior, again due to the limiter.

Table 1 also shows grid convergence slopes for $\nabla \cdot \boldsymbol{B}$ and Linde's quantity $F$ (Eq. (31)). For the first-order scheme, we see that $\nabla \cdot \boldsymbol{B}$ converges with an order not too far from the expected convergence order for state variables, although it is a quantity obtainable from the magnetic field through derivation. The reason may be that the numerical scheme treats $\nabla \cdot \boldsymbol{B} / \rho$ as a passive scalar [36]. Linde's quantity $F$ indeed converges one order faster than $\nabla \cdot \boldsymbol{B}$. The second-order scheme produces satisfactory grid convergence behavior for the $\nabla \cdot \boldsymbol{B}$-related quantities as well, except for the wedge, the bow shock and the nozzle. Again, the convergence degradation there can be traced back to errors at (not perfectly smooth) boundaries and shock-boundary interactions. The order we obtain for $\nabla \cdot \boldsymbol{B}$ convergence for our broad set of model problems is similar or higher than the order of convergence reported by Linde [31] and Barth [5] for smaller sets of model problems.

To conclude this section about the grid convergence study for global invariants, we can say that the experimentally obtained grid convergence behavior is satisfactorily consistent with the theoretically expected behavior. Some convergence degradation can be observed at (not perfectly smooth) boundaries and shock-boundary interactions, but this is not unexpected, given the discussion on convergence degradation in Section 5.6.1. The fluxes discussed in the previous section, which can be considered as one-time-integrated quantities, seem to be less sensitive to boundary effects. The results discussed in this section thus confirm again the validity of the numerical approach and the source term technique for controlling $\nabla \cdot \boldsymbol{B}$. 


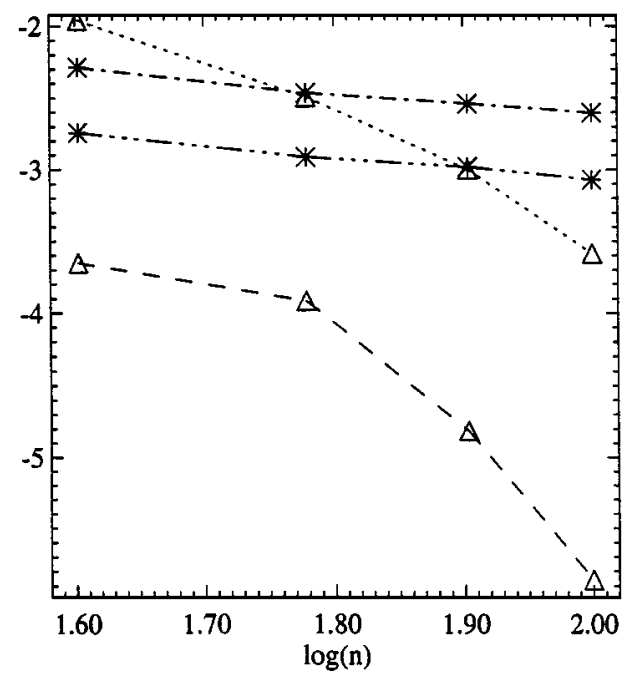

FIG. 12. The wedge flow. Grid convergence of the Rankine-Hugoniot relations. Ten logarithm of the relative errors of the downstream density and velocity at $(x=0.96, y=0.87)$, in function of the grid resolution. The density (first-order scheme, dotted, triangles, and second-order scheme, dashed, triangles) does converge, but not with a well-defined order. The velocity converges with $a=-0.79$ (first-order scheme, dash-dotted, asterisks) and with $a=-0.80$ (second-order scheme, dash-dot-dot-dotted, asterisks).

\subsection{Grid Convergence Results for Rankine-Hugoniot Relations}

For the wedge flow, the inflow quantities and the wedge angle completely determine the angle and the downstream quantities of the fast MHD shock. The algebraic equations of the MHD RH relations (6) can be solved numerically up to a very high accuracy, using for instance standard iterative methods provided in software for symbolical calculations. Following such a procedure, we have obtained the (nearly) exact downstream density and velocity field magnitude to be $\rho=2.00060295$ and $v=7.04956575$. In Fig. 12 we investigate grid convergence of the Rankine-Hugoniot relations. The base-10 logarithms of the relative errors of the downstream density and velocity at $(x=0.96, y=0.87)$ are shown, as functions of the grid resolution. First of all, we can say that our numerical scheme calculates a solution which closely matches the RH relations. The errors are $1 \%$ and lower. The velocity seems to converge linearly with a slope close to the theoretically predicted one, but the density seems to behave more erratically. Figure 13a indicates a reason for this. Finite-volume schemes have the well-known defect that they produce grid-related entropy oscillations in a direction parallel to a shock, and these entropy errors are advected downstream along the streamlines. This generates small ripples in the downstream solution, which should be uniform. The value of the downstream density, for instance, thus depends on the location and on the grid resolution, and this degrades pointwise convergence. The entropy oscillations are quite small with the Lax-Friedrichs scheme, and would be much larger when the Roe scheme was used. Due to the inherent defect of finite-volume schemes, we cannot prove proper grid convergence of the RH relations, but we can see that the RH relations are generally well satisfied and that the tendency is that they are better satisfied on finer grids. It will be interesting to see [11] if new MHD schemes based on multi-dimensional approaches will reduce or eliminate the downstream entropy contamination typical of finite volume approaches. 
(a) $s$ and streamlines

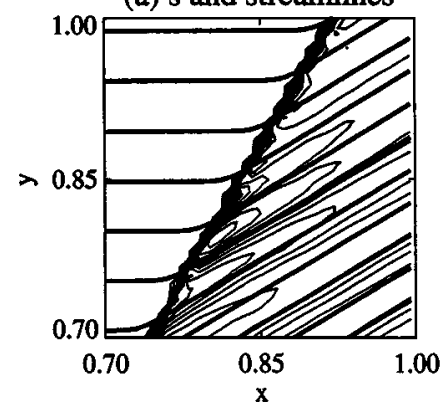

(b) $\operatorname{div}(B)$

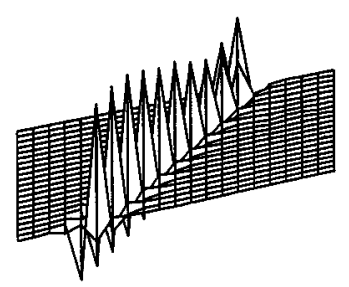

FIG. 13. The wedge flow. (a) Entropy contours (15 contours between $s=2.43$ and $s=2.45$, thin) and streamlines (thick) in a small region which contains the shock. Small errors in the entropy generated at the shock are convected away downstream parallel to the streamlines, generating small ripples in the downstream region which should be uniform. (b) Divergence of the B field in a small region containing the shock $(x \in[0.6,0.8], y \in$ $[0.55,0.75]) . \nabla \cdot \boldsymbol{B}$ is strongly non-zero in a small layer around the shock. Negative and positive $\nabla \cdot \boldsymbol{B}$ peaks cancel each other out, such that on a slightly more global scale, magnetic monopoles are not present. $\nabla \cdot \boldsymbol{B}$ reaches values from -3.36 to 6.76 in the region shown.

It is interesting here to investigate more closely what happens with the $\nabla \cdot \boldsymbol{B}$ constraint at a strong discontinuity. Figure $13 \mathrm{~b}$ shows the divergence of the $B$ field in a small region containing the shock $(x \in[0.6,0.8], y \in[0.55,0.75]) . \nabla \cdot \boldsymbol{B}$ is strongly non-zero in a small layer around the shock. $\nabla \cdot \boldsymbol{B}$ reaches values from -3.36 to 6.76 in the region shown. Actually, an upper bound for $\nabla \cdot \boldsymbol{B}$ can be given by $\nabla \cdot \boldsymbol{B}=|\Delta B| / \Delta x$, with $|\Delta B|$ the jump in the magnitude of the magnetic field vector across the shock, and $\Delta x$ a 1D measure of grid spacing. As in the MUSCL schemes discussed above, a stationary shock is generally captured with a constant number of intermediate cells which does not depend on the resolution, and as $|\Delta B|$ is independent of the resolution, this means that $\nabla \cdot \boldsymbol{B}$ peaks near shocks will grow without bounds as $1 / \Delta x$ in function of the grid resolution. As second-order schemes produce sharper shocks, the $\nabla \cdot \boldsymbol{B}$ peaks will be larger when a second-order scheme is used than when a first-order scheme is used, and our simulation results clearly confirm that (not shown). The Roe scheme, which produces sharper shocks than the Lax-Friedrichs scheme, also produces larger $\nabla \cdot \boldsymbol{B}$ peaks at shocks. The Powell source term approach takes these $\nabla \cdot \boldsymbol{B}$ peaks into account consistently and the source term precisely neutralizes the dynamical effect of the $\nabla \cdot \boldsymbol{B}$ peaks. It is important to note that the exact location and magnitude of the $\nabla \cdot \boldsymbol{B}$ peaks change when a different discretization is chosen for $\nabla \cdot \boldsymbol{B}$, and even in numerical schemes which guarantee $\nabla \cdot \boldsymbol{B}$ to vanish with machine precision in a certain discretization, it is clear that $\nabla \cdot \boldsymbol{B}$ peaks are present as soon as one looks at $\nabla \cdot \boldsymbol{B}$ in a different discretization. $\nabla \cdot \boldsymbol{B}$ peaks are an unavoidable consequence of our attempt to represent discontinuities on grids with a finite spatial resolution. All this seems to be quite worrisome, given that magnetic monopoles do not exist in nature, but in practice correct results seem to be produced by numerical schemes on discrete grids. How is this possible? As noted by Linde [31], discretization of $\nabla \cdot \boldsymbol{B}$ near shocks has a "telescoping" property, which can be described as follows. Negative and positive $\nabla \cdot \boldsymbol{B}$ peaks cancel each other out, such that on a slightly more global scale, magnetic monopoles are not present. This alternation of positive and negative $\nabla \cdot \boldsymbol{B}$ peaks can be seen clearly on Fig. 13. This is not yet a proof that the Powell approach produces valid results. Convincing support for the validity of Powell's source term approach is given by the results of our grid convergence studies. Table 1 shows that the measure of flux conservation $F$ converges faster than the state variables, and thus faster than predicted. Figure 12 shows that the numerical solution satisfies the MHD RH 


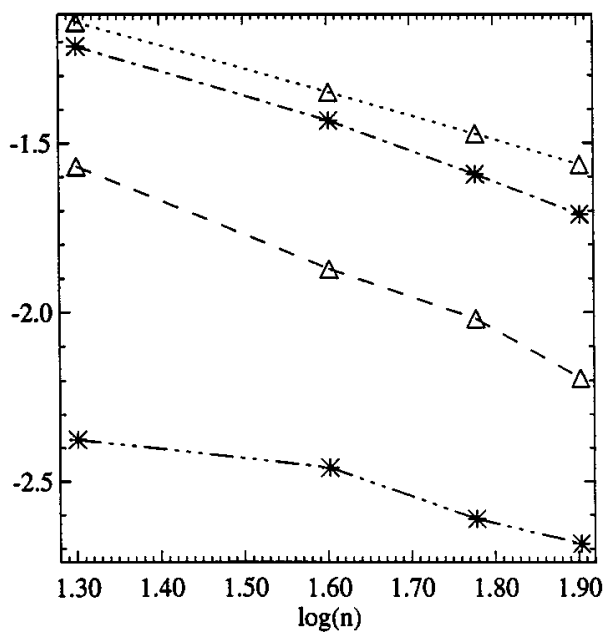

FIG. 14. The bow shock flow. Grid convergence of the stagnation point values. Ten logarithm of the relative errors of the stagnation point density and pressure, in function of the grid resolution. The density converges with $a=-0.70$ (first-order scheme, dotted, triangles), and with $a=-1.01$ (second-order scheme, dashed, triangles). The pressure converges with $a=-0.82$ (first-order scheme, dash-dotted, asterisks) and with $a=-0.52$ (secondorder scheme, dash-dot-dot-dotted, asterisks).

relations up to high precision. Most convincingly, Fig. 11 shows clearly that the magnetic flux through flux tubes which may contain fast shocks is conserved and that flux conservation converges with the theoretically predicted order.

For the bow shock flow, we can investigate grid convergence of the solution at the stagnation point. On the stagnation streamline, the RH relations lead to an analytical solution for the state variables downstream of the shock, and using the conservation of $s$ and $h_{s}$ on the stagnation streamline, the exact analytical solution of the stagnation point quantities in terms of the upstream flow quantities can be obtained [35]. For the inflow values of our bow shock model problem $\rho_{\text {stag }}=3.61528$ and $p_{\text {stag }}=3.61528$. Figure 14 shows grid convergence of the stagnation point values. Although the grid convergence orders are not all close to the theoretical value of one, there is a clear trend of grid convergence to the correct values, and this is a remarkable result, given the well-known problems of many finite-volume schemes with "wall-heating" at perfect walls [29].

\section{SUMMARY AND CONCLUSION}

In this paper we have started out with a brief presentation of the theory of stationary characteristics for the class of 2D planar field-aligned MHD flows. We have presented five model flows of increasing complexity belonging to this class, and we have investigated the physical properties of these flows using characteristic theory. We have used characteristic theory to formulate grid convergence criteria for flows belonging to this class, and we have shown grid convergence for the numerical simulation of the five field-aligned model flows and for one non-field-aligned model flow with a standard high-resolution finite volume numerical MHD code on structured body-fitted grids.

We did not always obtain grid convergence orders completely consistent with theoretical expectations. Such degradation of convergence can mostly be attributed to analytical singularities or numerical inaccuracies at boundaries, to complex behavior at discontinuities, and to the distortion of the grid. However, in general we did obtain satisfactory grid 
convergence for most properties. The conservation of magnetic and momentum flux in flux tubes behaved especially well (see Table 1). All these results give us strong indications that we calculate the physically correct solution to the flow problems and that we improve the accuracy by refining the grid. It is important to establish such formal accuracy tests for MHD simulations, and this is certainly not trivial given the lack of analytical solutions for 2D MHD problems. More advanced schemes and boundary treatment may lead to more completely consistent grid convergence results, and throughout this paper we have indicated in which direction improvement can be expected.

We have investigated by formal grid convergence studies of magnetic flux conservation and other flow quantities whether the Powell source term approach to control the $\nabla \cdot \boldsymbol{B}$ constraint leads to correct results for this class of flows. Our grid convergence results show clearly that this method leads to correct solutions for the transonic problems we considered, although it remains difficult to grasp all the subtle details of how this correct result is obtained. It may also be that for some problems, for which the conservation of magnetic flux up to very high accuracy is crucial, the source term technique would turn out to be insufficient. Recently Toth [55] has given an example of a time-dependent flow (a strong Riemann problem) for which the source term approach does not seem to work satisfactorily.

Although the model problems presented in this article exhibit a variety of flow features, they all belong to the sub-class of stationary planar 2D MHD flows. Stationary flow has important applications, and in this paper we have taken a first step to prove grid convergence for model problems belonging to this important sub-class of flows. It would certainly be useful to develop more general test problems allowing for grid convergence studies, which, for instance, would also investigate the accuracy of time integration. Self-similar MHD flows are probably good candidates for this. Fully 3D test problems should be considered as well. In 3D, when the magnetic field is not aligned to the flow, many of the invariants used in this paper to prove grid convergence cease to be invariant [46], so it is to be expected that it will be more difficult to formulate 3D MHD model flow problems which allow for grid convergence study. For the time being, however, we can reassure ourselves by realizing that many 3D algorithms are straight extensions of their 2D counterparts, so it can be expected that the $3 \mathrm{D}$ algorithms perform similarly to the $2 \mathrm{D}$ algorithms in terms of accuracy.

\section{ACKNOWLEDGMENTS}

H.D.S. acknowledges illuminating discussions with B. C. Low, K. MacGregor, K. Powell, G. Toth, and T. Barth.

\section{REFERENCES}

1. D. Vanden Abeele and H. Deconinck, Development of a Godunov-Type Solver for 2D ideal MHD Problems, Technical Report 13 (von Karman Institute for Fluid Dynamics, 1995).

2. J. Edward Anderson, Magnetohydrodynamic Shock Waves, Ph.D. thesis (M. I. T., 1963).

3. D. S. Balsara and D. S. Spicer, A staggered mesh algorithm using high order Godunov fluxes to ensure solenoidal magnetic fields in magnetohydrodynamic simulations, J. Comput. Phys. 149, 270 (1999).

4. A. A. Barmin, A. G. Kulikovskiy, and N. V. Pogorelov, Shock-capturing approach and nonevolutionary solutions in magnetohydrodynamics, J. Comput. Phys. 126, 77 (1996).

5. Timothy J. Barth, Numerical methods for gasdynamic systems on unstructured meshes, in An Introduction to Recent Developments in Theory and Numerics for Conservation Laws, edited by D. Kröner, M. Ohlberger, and C. Rohde, Lecture Notes in Computational Science and Engineering (Springer-Verlag, Berlin, 1998), p. 195.

6. D. Biskamp, Nonlinear Magnetohydrodynamics (Cambridge Univ. Press, Cambridge, UK, 1993). 
7. J. U. Brackbill and D. C. Barnes, The effect of nonzero $\nabla \cdot \boldsymbol{B}$ on the numerical solution of the magnetohydrodynamic equations, J. Comput. Phys. 35, 426 (1980).

8. M. Brio and C. C. Wu, An upwind differencing scheme for the equations of ideal magnetohydrodynamics, J. Comput. Phys. 75, 400 (1988).

9. R. Courant and K. O. Friedrichs, Supersonic Flow and Shock Waves (Interscience, New York, 1948).

10. R. Courant and D. Hilbert, Methods of Mathematical Physics, Vol. 2 (Interscience, New York, 1962).

11. A. Csik, H. Deconinck, and S. Poedts, Monotone residual distribution schemes for the ideal 2D magnetohydrodynamic equations on unstructured grids, AIAA Paper 99-3325-CP (1999).

12. W. Dai and P. R. Woodward, An approximate Riemann solver for ideal magnetohydrodynamics, J. Comput. Phys. 111, 354 (1994).

13. W. Dai and P. R. Woodward, Extension of the piecewise parabolic method (PPM) to multidimensional ideal magnetohydrodynamics, J. Comput. Phys. 115, 485 (1994).

14. W. Dai and P. R. Woodward, On the divergence-free condition and conservation laws in numerical simulations for supersonic magnetohydrodynamic flows, Astrophys. J. 494, 317 (1998).

15. C. R. DeVore, Flux-corrected transport techniques for multidimensional compressible magnetohydrodynamics, J. Comput. Phys. 92, 142 (1991).

16. C. R. Evans and J. F. Hawley, Simulation of magnetohydrodynamic flows: A constrained transport method, Astrophys. J. 332, 659 (1988).

17. S. A. E. G. Falle, S. S. Komissarov, and P. Joarder, A multidimensional upwind scheme for magnetohydrodynamics, Mon. Not. R. Astron. Soc. 297, 265 (1998).

18. S. K. Godunov, In Nonlinear Hyperbolic Problems: Proceedings of an Advanced Research Workshop, Vol. 1270 of Lecture Notes in Mathematics, edited by C. Carasso, P.-A. Raviart, and D. Serre (SpringerVerlag, Berlin, 1987).

19. J. P. Goedbloed and A. Lifshitz, Stationary symmetric magnetohydrodynamic flows, Phys. Plasmas 4, 3544 (1997)

20. T. I. Gombosi, K. G. Powell, and D. L. De Zeeuw, Axisymmetric modeling of cometary mass loading on an adaptively refined grid: MHD results, J. Geophys. Res. 99, 21525 (1994).

21. T. I. Gombosi, D. L. De Zeeuw, R. L. Haberli, and K. G. Powell, Three-dimensional multiscale MHD model of cometary plasma environments, J. Geophys. Res. 101, 15233 (1996).

22. H. Grad, Reducible problems in magneto-fluid dynamic steady flows, Rev. Mod. Phys. 32, 830 (1960).

23. E. Hameiri, The equilibrium and stability of rotating plasmas, Phys. Fluids 26, 230 (1983).

24. A. Jeffrey and T. Taniuti, Non-linear Wave Propagation (Academic Press, New York, 1964).

25. Y. Kato and T. Taniuti, Hydromagnetic plane steady flow in compressible ionized gases, Prog. Theor. Phys. 21, 606 (1959).

26. R. Keppens and J. P. Goedbloed, Numerical simulations of stellar winds: Polytropic models, Astron. Astrophys. 343, 251 (1998).

27. L. D. Landau and E. M. Lifshitz, Electrodynamics of Continuous Media (Pergamon, Oxford, 1984).

28. R. J. Leveque, Numerical Methods for Conservation Laws, Lectures in Mathematics ETH Zurich (Birkhauser-Verlag, Basel, 1992).

29. R. J. Leveque, Nonlinear conservation laws and finite volume methods for astrophysical fluid flow, in Computational Methods for Astrophysical Fluid Flow, edited by O. Steiner and A. Gautschy (Springer-Verlag, Berlin, 1998).

30. A. Lifshitz and J. P. Goedbloed, Transonic magnetohydrodynamic flows, J. Plasma Physics 58, 61 (1997).

31. T. J. Linde, A Three-Dimensional Adaptive Multifluid MHD Model of the Heliosphere, Dissertation (Univ. of Michigan, 1998).

32. T. J. Linde, T. I. Gombosi, P. L. Roe, K. G. Powell, and D. L. De Zeeuw, Heliosphere in the magnetized local interstellar medium: Results of a three-dimensional MHD simulation. J. Geophys. Res. 103, 1889 (1998).

33. M. Manna, A Three Dimensional High Resolution Compressible Flow Solver, Dissertation (Universite Catholique de Louvain, 1992).

34. R. S. Myong and P. L. Roe, On Godunov-type schemes for magnetohydrodynamics. 1. A model system, $J$. Comput. Phys. 147, 545 (1998). 
35. S. M. Petrinec and C. T. Russell, Hydrodynamic and MHD equations across the bow shock and along the surfaces of planetary obstacles, Space Sci. Rev. 79, 757 (1997).

36. K. G. Powell, P. L. Roe, R. S. Myong, T. I. Gombosi, and D. L. De Zeeuw, An Upwind Scheme for Magnetohydrodynamics, AIAA Paper 95-1704-CP (1995); K. G. Powell, P. L. Roe, T. J. Linde, T. I. Gombosi, and D. L. De Zeeuw, A solution-adaptive upwind scheme for ideal magnetohydrodynamics, J. Comput. Phys. 154, 284 (1999).

37. James J. Quirk, A contribution to the great Riemann solver debate, Int. J. Numer. Methods Fluids 18, 555 (1994).

38. R. Ratkiewicz, A. Barnes, G. A. Molvik, J. R. Spreiter, S. S. Stahara, M. Vinokur, and S. Venkateswaran, Effect of varying strength and orientation of local interstellar magnetic field on configuration of exterior heliosphere: 3D MHD simulations, Astron. Astrophys. 335, 363 (1998).

39. P. L. Roe and D. S. Balsara, Notes on the eigensystem of magnetohydrodynamics, SIAM J. Appl. Math. 56, 57 (1996).

40. D. Ryu and T. W. Jones, Numerical magnetohydrodynamics in astrophysics: Algorithm and tests for one-dimensional flow, Astrophys. J. 442, 228 (1995).

41. D. Ryu, T. W. Jones, and A. Frank, Numerical magnetohydrodynamics in astrophysics: Algorithm and tests for multidimensional flow, Astrophys. J. 452, 785 (1995).

42. T. Sakurai, Magnetohydrodynamic solar/stellar wind models, Comput. Phys. Rep. 12, 247 (1990).

43. C. Schwab, hp-fem for fluid flow simulation, in High Order Discretization Methods in Computational Fluid dynamics, edited by H. Deconinck and T. J. Barth, von Karman Institute Lecture Series (1998).

44. H. De Sterck, H. Deconinck, S. Poedts, and D. Roose, A bow shock flow containing (almost) all types of ('exotic') MHD discontinuities, in Proceedings of the Seventh International Conference on Hyperbolic Problems (Birkhauser-Verlag, Basel, 1998), p. 195.

45. H. De Sterck, B. C. Low, and S. Poedts, Complex magnetohydrodynamic bow shock topology in field-aligned low- $\beta$ flow around a perfectly conducting cylinder, Phys. Plasmas 5, 4015 (1998).

46. H. De Sterck, B. C. Low, and S. Poedts, Characteristic analysis of a complex two-dimensional magnetohydrodynamic bow shock flow with steady compound shocks, Phys. Plasmas 6, 954 (1999).

47. H. De Sterck and S. Poedts, Intermediate shocks in three-dimensional magnetohydrodynamic bow shock flows with multiple interacting shock fronts, Phys. Rev. Lett. 84, 5524 (2000).

48. H. De Sterck, S. Poedts, and J. P. Goedbloed, Dynamics of hot filaments in a tokamak plasma, J. Plasma Phys. 59, 277 (1998).

49. J. M. Stone, J. F. Hawley, C. R. Evans, and M. L. Norman, A test suite for magnetohydrodynamical simulations, Astrophys. J. 388, 415 (1992).

50. J. M. Stone and M. L. Norman, ZEUS-2D: A radiation magnetohydrodynamics code for astrophysical flows in two space dimensions. ii. The magnetohydrodynamic algorithms and tests, Astrophys. J. Suppl. 80, 791 (1992).

51. T. Tanaka, Configurations of the solar wind flow and magnetic field around the planets with no magnetic field: Calculation by a new MHD simulation scheme, J. Geophys. Res. 98, 251 (1993).

52. T. Tanaka, Finite volume TVD scheme on an unstructured grid system for three-dimensional MHD simulations of inhomogeneous systems including strong background potential field, J. Comput. Phys. 111, 381 (1994).

53. P. A. Thompson, Compressible-Fluid Dynamics (McGraw-Hill, New York, 1972).

54. G. Toth, R. Keppens, and M. A. Botchev, Implicit and semi-implicit schemes in the Versatile Advection Code: Numerical tests, Astron. Astrophys. 332, 1159 (1998).

55. G. Toth and D. Odstrcil, Comparison of some Flux Corrected Transport and Total Variation Diminishing numerical schemes for hydrodynamic and magnetohydrodynamic problems, J. Comput. Phys. 128, 82 (1996); G. Toth, The $\nabla \cdot B=0$ constraint in shock-capturing magnetohydrodynamics codes, J. Comput. Phys. 161, 605 (2000).

56. E. Turkel, Accuracy of Schemes with Non-uniform Meshes for Compressible Fluid Flows, Technical Report 85-43 (ICASE Langley, VA, 1985).

57. B. van Leer, Towards the ultimate conservative difference scheme. V. A second order sequel to Godunov's method, J. Comput. Phys. 32, 101 (1979).

58. A. L. Zachary, A. Malagoli, and P. Colella, A higher-order method for multidimensional ideal magnetohydrodynamics, SIAM J. Sci. Comput. 15, 263 (1994). 